\title{
THE DISTRIBUTION OF PERIODIC TORUS ORBITS ON HOMOGENEOUS SPACES
}

\author{
MANFRED EINSIEDLER, ELON LINDENSTRAUSS, PHILIPPE MICHEL AND AKSHAY \\ VENKATESH
}

\begin{abstract}
We prove results towards the equidistribution of certain families of periodic torus orbits on homogeneous spaces, with particular focus on the case of the diagonal torus acting on quotients of $\mathrm{PGL}_{n}(\mathbb{R})$. After attaching to each periodic orbit an integral invariant (the discriminant) our results have the following flavour: certain standard conjectures about the distribution of such orbits hold up to exceptional sets of at most $O\left(\Delta^{\epsilon}\right)$ orbits of discriminant $\leq \Delta$. The proof relies on the well-separatedness of periodic orbits together with measure rigidity for torus actions. We also give examples of sequences of periodic orbits of this action that fail to become equidistributed, even in higher rank.

We give an application of our results to sharpen a theorem of Minkowski on ideal classes in totally real number fields of cubic and higher degrees.
\end{abstract}

\section{Contents}

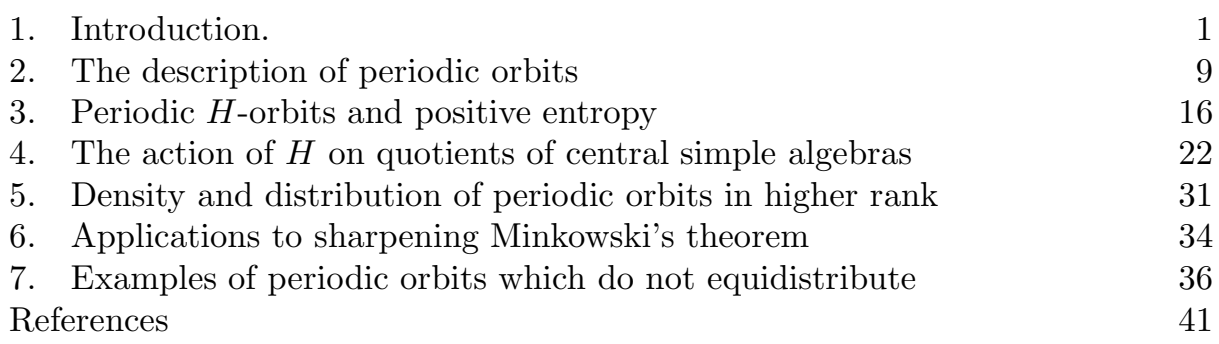

\section{INTRODUCTION.}

1.1. Periodic torus orbits. Let $G=\mathbf{G}(\mathbb{R})$ be a real algebraic group defined over $\mathbb{Q}$ (later we will specialize to the case of $\mathbb{R}$-split groups), and $\Gamma<G$ an arithmetic lattice. ${ }^{1}$ Let $L$ be a closed subgroup of $G$. We say that an $L$-orbit on $\Gamma \backslash G$ is periodic if it possesses a finite $L$-invariant measure ${ }^{2}$. Periodic orbits of subgroups $L<G$ on such arithmetic quotients have been studied in various context by several authors,

Date: $7 / 27 / 2006$.

This research has been supported by the Clay Mathematics Institute (M.E. by a Clay Research Scholarship, E.L. and A.V. by fellowships), by the "Réseau arithmétique des Pyrénées" of Région Languedoc-Roussillon and the European TMR Network "Arithmetic Algebraic Geometry" (Ph.M.); and by the NSF (DMS grants 0509350 (M.E.), 0500205 (E.L.), 02045606 (A.V.) and an FRG collaborative grant.)

${ }^{1}$ I.e. a lattice in $G$ contained in $\mathbf{G}(\mathbb{Q})$.

${ }^{2}$ This is not a completely standard definition, but is quite natural. 
and have proved to be a fruitful meeting ground of dynamics and number theory. We note that there is nothing special about real algebraic groups: we could have equally well considered $p$-adic or $S$-adic ${ }^{3}$ groups. We focus on real groups only to simplify matters and for concreteness of exposition.

The multiple facets of the study of periodic orbits on arithmetic quotients can already be seen in the simple but important case of $\mathbf{G}=\mathrm{PGL}_{2}$. In this case, it is well-known that the following objects are essentially in bijection:

(1) Closed geodesics on the modular surface $\mathrm{SL}_{2}(\mathbb{Z}) \backslash \mathbb{H}$.

(2) Periodic orbits of the diagonal subgroup of $\mathrm{PGL}_{2}(\mathbb{R})$ on the arithmetic quotient $\mathrm{PGL}_{2}(\mathbb{Z}) \backslash \mathrm{PGL}_{2}(\mathbb{R})$.

(3) Ideal classes in real quadratic orders (e.g. $\mathbb{Z}[\sqrt{d}]$ for $d>0$ ).

A natural question one is led to, when studying periodic $L$-orbits on arithmetic quotients, is the following:

Basic Question: to what extent do larger and larger periodic L-orbits fill out more and more of $\Gamma \backslash G$ ?

If the group $L$ is generated by its unipotent ${ }^{4}$ elements, Ratner's measure classification theorem [37], together with a "linearization" technique developed in the work of Ratner [38, Dani-Margulis [6], Shah [43] and elsewhere, can be used to give an essentially complete answer to the basic question above: larger and larger periodic $L$-orbits become equidistributed in $\Gamma \backslash G$ unless there are certain obvious obstacles present (see e.g. [15] and [31] for such results with arithmetic consequences).

Our aim in this paper and its sequels (currently two are planned) is to study periodic orbits of a maximal $\mathbb{R}$-split torus $H<G$ on $G / \Gamma$ for $G=\mathbf{G}(\mathbb{R})$ an $\mathbb{R}$-split group. Such groups $H$ have by definition no unipotent elements whatsoever, and their action is much less well-understood ${ }^{5}$. While much of our discussion is quite general, we focus in the introduction and the later sections of this paper on the following two concrete examples:

$(\mathrm{L}-1) \mathbf{G}=\mathrm{PGL}_{n}, G=\mathrm{PGL}_{n}(\mathbb{R}), \Gamma=\mathrm{PGL}_{n}(\mathbb{Z})$

$(\mathrm{L}-2) D_{\mathbb{Q}}$ a degree $n$ division algebra over $\mathbb{Q}$, with $D_{\mathbb{Q}} \otimes_{\mathbb{Q}} \mathbb{R} \cong M_{n}(\mathbb{R})$, G the group associated to invertible elements of $D$ modulo center, $G=\mathbf{G}(\mathbb{R}) \cong$ $\mathrm{PGL}_{n}(\mathbb{R})$ and $\Gamma<G$ the lattice associated with an order $\mathcal{O}_{D}$ in $D_{\mathbb{Q}} \cdot{ }^{6}$

Identifying $G \cong \mathrm{PGL}_{n}(\mathbb{R})$, in both cases we can take $H$ to be the group of $n \times$ $n$ diagonal matrices ${ }^{7}$. Both examples come from a common family of lattices in $\mathrm{PGL}_{n}(\mathbb{R})$ arising from central simple algebras; in the first case $\Gamma \backslash G$ is not compact, in the second it is.

1.2. Discriminant, shape and volume of periodic orbits. Let $H$ be an $\mathbb{R}$-split maximal torus in $G$ as above ${ }^{8}$. Note that since $H$ is abelian, an orbit $x H=\Gamma g H$ of $H$ is periodic if and only if $x H$ is a compact subset of $\Gamma \backslash G$.

\footnotetext{
${ }^{3}$ I.e. products of real and $p$-adic groups (possibly for several different primes $p$ ).

${ }^{4} \mathrm{An}$ element $g$ of a (linear) algebraic group $\mathbf{G}(K)$ is said to be unipotent if for some faithful representation $\rho$ of $\mathbf{G}$, all eigenvalues of $\rho(g)$ over the algebraic closure of $K$ are equal to 1 .

${ }^{5}$ At least for $\operatorname{dim} H \geq 2$.

${ }^{6}$ See $\$ 4.1$ for a more complete discussion.

${ }^{7}$ Considered as elements in $\mathrm{PGL}_{n}(\mathbb{R})$, i.e. with proportional matrices identified.

${ }^{8} \mathrm{~A}$ maximal torus in $G$ is also called a Cartan subgroup, and we shall use the two terms interchangeably.
} 
To such a periodic $H$-orbit we can attach several invariants. For example, we can look at the "shape" of the orbit, i.e. the stabilizer $g^{-1} \Gamma g \cap H$ of $x$, which is a lattice in $H$. In particular, fixing a Haar measure on $H$, we can consider the volume (or regulator) of the periodic orbit $x H$, which by definition is the covolume of $g^{-1} \Gamma g \cap H$ in $H$. We obtain another less obvious but nontheless important invariant of the periodic orbit, the discriminant of $x H$ (denoted $\operatorname{disc}(x H)$ ), by associating to each periodic orbit a rational point on an appropriate variety and looking at the denominator of this point. By definition the discriminant is always a positive integer.

It turns out that the relative sizes of the discriminant and regulator of an orbits play a crucial role in the study of these orbits. In general, discriminant and regulator satisfy the following relations (see Proposition 2.8 below): there is some $c$ (which can be taken to be $1 / 2+\epsilon$ for the arithmetic quotients given in (L-1) and (L-2) above) so that for any periodic $H$-orbit $x H$,

$$
\log \operatorname{disc}(x H) \ll \operatorname{vol}(x H) \ll \operatorname{disc}(x H)^{c}
$$

Our results, as well as the examples we give in Section 7, all give credence to the general principle that the bigger the volume of a periodic orbit relative to its discriminant, the better the orbit is behaved.

By considering periodic orbits individually, as we do in this paper, one does not take into account an important hidden symmetry of the problem. Returning to the example of periodic $H$-orbits on $\mathrm{PGL}_{2}(\mathbb{Z}) \backslash \mathrm{PGL}_{2}(\mathbb{R})$, the "volume" of a periodic orbit is simply its length, or equivalently the length of the corresponding closed geodesics on $\mathrm{SL}(2, \mathbb{Z}) \backslash \mathbb{H}$, and the discriminant coincides with the discriminant of the associated real quadratic other. As is well-known, the length spectrum of closed geodesics in this case is far from simple.

A similar property holds for all $n$, both for $\Gamma=\mathrm{PGL}_{n}(\mathbb{Z})$ and more general lattices constructed from central simple algebras and division algebras. The periodic $H$ orbits naturally come in packets, with all orbits in a packet sharing the same discriminant, regulator, and even shape. These packets can be understood as projections to $\Gamma \backslash G$ of orbits of adelic $\mathbb{Q}$-tori on $\mathbf{G}(\mathbb{Q}) \backslash \mathbf{G}(\mathbb{A})$. The compact orbits belonging to a single packet are therefore parameterized by a finite abelian group - a suitable class group.

One important property of these packets is that their total volume (which is simply the volume of any single orbit in the packet times the number of such periodic orbits) is equal to $D^{1 / 2+o(1)}$ where $D$ is the discriminant of the packet i.e. essentially equal to the upper bound in (1.1). These packets are considered in detail in 12, 14. A coarser grouping of periodic orbits can be obtained by using Hecke correspondences, and this is analyzed in [1].

1.3. Conjectures on higher rank rigidity. The dynamics of the group $H$ is drastically different in the rank one and higher rank cases. In the rank one case, for example $\mathrm{PGL}_{2}(\mathbb{Z}) \backslash \mathrm{PGL}_{2}(\mathbb{R})$, the closing lemma assures us that each periodic orbit individually can be distributed in an almost arbitrary way; the situation is very different, however, if one considers packets of periodic orbits as will be explained below. 
In the higher rank cases, the dynamics is much more rigid. For example, G. A. Margulis has highlighted the following conjecture, which is equivalent to a conjecture about the values of products of linear forms formulated by Cassels and Swinerton-Dyer in 1955 [5]:

Conjecture 1.1. Let $H$ be the subgroup of diagonal matrices in $\mathrm{PGL}_{n}(\mathbb{R})$ for $n \geq 3$. Any bounded ${ }^{9} H$-orbit in $\mathrm{PGL}_{n}(\mathbb{Z}) \backslash \mathrm{PGL}_{n}(\mathbb{R})$ is closed.

There are also related conjectures regarding invariant measures, due to H. Furstenberg (unpublished), A. Katok and R. Spatzier [20], and G. A. Margulis [30; a fairly general variant of these conjectures can be found in [12, Conjecture 2.4]. Concretely one expects the following:

Conjecture 1.2. Let $n \geq 3, G \cong \mathrm{PGL}_{n}(\mathbb{R}), \Gamma<G$ a lattice as in examples (L-1) or (L-2) above ${ }^{10}$. Let $\mu$ be an $H$-invariant and ergodic measure. Then $\mu$ is homogeneous: i.e. there is a closed group $L \leq G$ so that $\mu$ is an L-invariant measure on a single L-orbit.

Given these conjectures, it is reasonable to expect that periodic orbits of $H$ are nicely distributed in $\Gamma \backslash G$. Indeed, as remarked by Margulis, the following follows from Conjecture 1.1 and the isolation results of [5]:

Conjecture 1.3. Let $n \geq 3$. For any compact $\Omega \subset \mathrm{PGL}_{n}(\mathbb{Z}) \backslash \mathrm{PGL}_{n}(\mathbb{R})$ there are only finitely many periodic $H$-orbits contained in $\Omega$.

\subsection{Distribution and density of periodic orbits: statement of main re- sults.}

1.4.1. Periodic orbits and their approach to infinity, in the noncompact case. We obtain the following towards Conjecture 1.3 .

Theorem 1.4. Let $n \geq 3$. For any compact $\Omega \subset \operatorname{PGL}_{n}(\mathbb{Z}) \backslash \operatorname{PGL}_{n}(\mathbb{R})$ and any $\varepsilon>0$ the total volume of all periodic $H$-orbits contained in $\Omega$ of discriminant $\leq D$ is $\ll_{\varepsilon, \Omega} D^{\varepsilon}$.

In contrast, for $n=2$ we have the following:

Theorem 1.5. For every $\varepsilon>0$ there is a compact $\Omega \subset \mathrm{PGL}_{2}(\mathbb{Z}) \backslash \mathrm{PGL}_{2}(\mathbb{R})$ so that the total length of all periodic $H$-orbits contained in $\Omega$ of discriminant $\leq D$ is $\gg_{\epsilon} D^{1-\epsilon}$.

Further, even for $n \geq 3$, individual orbits may fail to become equidistributed, and, indeed, spend a positive fraction of their time "at $\infty$ ", at least asymptotically - see Section 1.5 .

1.4.2. Density and distribution in the compact case. In the following results, let $\Gamma, G$ be as in (L-2) and $n \geq 3$.

Given a finite collection $Y=\left\{x_{i} H: 1 \leq i \leq l\right\}$ of periodic orbits, we define

$$
\operatorname{disc}(Y):=\max _{1 \leq i \leq l} \operatorname{disc}\left(x_{i} H\right), \quad \operatorname{vol}(Y):=\sum_{i=1}^{l} \operatorname{vol}\left(x_{i} H\right) .
$$

\footnotetext{
${ }^{9}$ I.e. an orbit with compact closure.

${ }^{10}$ More generally, any lattice coming from a central simple algebra over $\mathbb{Q}$; examples due to Rees 39] (a more accessible source is [9, Section 9]) show that in order to cover more general (even cocompact) lattices in $G$ the conjecture should be reformulated to allow for rank one factors (see e.g. [30] or [12]).
} 
Given such a collection $Y$, we let $\mu_{Y}$ be the sum of volume measures on $x_{i} H$, normalized to have total mass 1 .

Theorem 1.6. Let $n \geq 3$ and let $\rho>0$ be fixed. For each $j \geq 1$, let $Y_{j}$ be a finite collection of periodic $H$-orbits satisying $\operatorname{disc}\left(Y_{j}\right) \rightarrow \infty$ and $\operatorname{vol}\left(Y_{j}\right) \geq \operatorname{disc}\left(Y_{j}\right)^{\rho}$. Suppose there is no periodic orbit of a group $H<L<G$ (with both inclusions proper) containing infinitely many periodic orbits belonging to $\bigcup Y_{j}$. Then $\overline{\bigcup x_{j} H}=$ $\Gamma \backslash G$.

Our precise distribution result is stated in Theorem 5.1, but is rather complicated in general because of the existence of intermediate subgroups. For now we simply state a corollary of that theorem:

Corollary 1.7. (to Theorem 5.1). Let $\Gamma, G$ be as in (L-2) with $n$ prime, and let $\rho>0$ be fixed.

For each $j \geq 1$, let $Y_{j}$ be a finite collection of periodic $H$-orbits satisfying $\operatorname{disc}\left(Y_{j}\right) \rightarrow \infty$ and $\operatorname{vol}\left(Y_{j}\right)>\operatorname{disc}\left(Y_{j}\right)^{\rho}$. Then any weak limit $\mu$ of the $\mu_{Y_{j}}$ satisfies

$$
\mu(B) \geq c_{n} . \rho \cdot \mu_{\text {Haar }}(B),
$$

for any measurable set $B \subset X$; here $\mu_{\text {Haar }}$ is the $G$-invariant (Haar) probability measure on $\Gamma \backslash G(\mathbb{R})$ and $c_{n}$ is a positive constant which depends on $n$ only.

Example 1.8. A sequence $\left\{Y_{j}\right\}_{j \geq 1}$ to which this corollary applies is the following: let $V_{1}<V_{2}<\cdots<V_{j}, \ldots$ denote the "volume spectrum" of $X$ (i.e. the sequence of the volumes of all periodic $H$-orbits in increasing order), and let $Y_{j}$ be the collection of all orbits of volume $V_{j}$. It can be shown that the hypothesis of Corollary 1.7 is satisfied for any $\rho<1 / 2$ and $j$ large enough.

More generally, we can rephrase the corollary in the following way. Fixing a subset $U \subset \Gamma \backslash G(\mathbb{R})$, we call a periodic $H$-orbit $(U, \varepsilon)$-bad if the compact orbit spends less than $\varepsilon \mu_{\text {Haar }}(U)$ time inside $U$. Then there is an constant $c_{n}>0$, depending only on $n$, so that the total volume of $(U, \varepsilon)$-bad orbits of discriminant $\leq D$ is $\ll_{\varepsilon, U} D^{\varepsilon c_{n}}$. In particular, the total volume of periodic orbits that fail to intersect $U$ and have discriminant $\leq D$ is $\ll_{\varepsilon, U} D^{\varepsilon}$.

1.4.3. Linnik's principle. The key observation behind Theorem 1.4 and Theorem 1.6 is the following relation between total volume of a collection of periodic orbits in the relation to their discriminants and the entropy of any weak* limit. In his book [26], Yu. Linnik discusses several equidistribution problems, among them the equidistribution of packets of periodic geodesic trajectories on $\mathrm{PGL}_{2}(\mathbb{Z}) \backslash \mathrm{PGL}_{2}(\mathbb{R})$. Central to the proof of each of these equidistribution statements is a "basic lemma" which can be viewed as an implicit form of such a relation between volume and entropy, and so we call this general phenomenon Linnik's Principle.

Theorem 1.9 (Linnik's Principle). For $i \geq 1$, let $Y_{i}$ be a collection of periodic $H$-orbits in $X=\Gamma \backslash G$ satisfying $\operatorname{disc}\left(Y_{i}\right) \rightarrow \infty$ and $\operatorname{vol}\left(Y_{i}\right) \geq \operatorname{disc}\left(Y_{i}\right)^{\rho}$, for some fixed $\rho>0$. Let $\mu_{i}=\mu_{Y_{i}}$ be the probability measure associated with $Y_{i}$. Suppose that $\mu_{i} \rightarrow \mu$ as $i \rightarrow \infty$ in the weak* topology for some probability measure $\mu$. Then for any regular $h \in H$ there is an explicit $c_{h}>0$ (depending only on $h$ ) so that

$$
h_{\mu}(h) \geq c_{h} \rho .
$$


1.5. Counterexamples to equidistribution. Theorems 1.4 and 1.6 as well as Corollary 1.7 fall far short of one possible candidate for an answer to the basic question of Section 1.1: that possibly periodic $H$-orbits of increasing volume which do not lie in any periodic orbits of a bigger group ${ }^{11}$ become equidistributed in $\Gamma \backslash G$. This is not just because of technical difficulties; indeed, this plausible statement regarding equidistribution of $H$-orbits is false, and the number of periodic $H$-orbits which fail to be equidistributed is asymptotically bigger than $D^{\alpha}$ for some strictly positive $\alpha$ :

Theorem 1.10. Let $n \geq 2$. There is a sequence of periodic $H$-orbits $x_{i} H$ on the space $\mathrm{PGL}_{n}(\mathbb{Z}) \backslash \mathrm{PGL}_{n}(\mathbb{R})$ and $\alpha, \delta>0$ such that

(1) any weak* limit of the probability measures $\mu_{x_{i} H}$ supported on these periodic $H$-orbits has total mass $<1-\delta$.

(2) the total volume $\operatorname{vol}\left(\left\{x_{i} H: \operatorname{disc}\left(x_{i} H\right)<D\right\}\right) \gg D^{\alpha}$.

This theorem is proved by an explicit construction (using a construction of Duke [8) suggested to us by P. Sarnak; a closely related construction in the special case of $n=2$ can be found in [42].

One might hope escape of mass to infinity is the only possible obstacle to equidistribution of periodic $H$-orbits, and that things are nicer in the compact case. For example, one might hope that Corollary 1.7 could be sharpened to give equidistribution, i.e. that the limiting measure $\mu=\mu_{\text {Haar }}$. Even this seems highly unlikely.

While we do not have a counterexample in this particular setting (i.e. $G \cong$ $\operatorname{PGL}(n, \mathbb{R})$ and $\Gamma$ as in (L-2), with $n \geq 3)$ we believe the following is likely: that for any periodic $H$-orbits $x_{0} H$ in $\Gamma \backslash G$, there should exists collections $Y_{i}$ of periodic $H$-orbits with discriminants in the range $\left[\Delta_{i}, 2 \Delta_{i}\right]$ and with $\operatorname{vol}\left(Y_{i}\right) \gg \Delta_{i}^{\alpha}$ so that $\mu_{i}$ converge weak* to a measure $\mu$ assigning positive measure to the given periodic $H$-orbit $x_{0} H$. Moreover, we give an explicit construction of this type in the $S$ arithmetic context in [13].

Besides showing the limit of what one may hope to prove regarding periodic torus orbits, these examples also point out to an important difference between torus actions and the action of groups generated by unipotents: linearization techniques à la Ratner, Dani-Margulis and Shah do not work for torus actions, at least not on the level of individual periodic orbits, and moreover any substitute for these techniques will either have to assume much more or give much less ${ }^{12}$.

1.6. A conjecture. Evidence from harmonic analysis. The construction of nonequidistributing periodic orbits described in Section 1.5 involve orbits $x_{i} H$ that have very small volume relative to their discriminant: $\operatorname{vol}\left(x_{i} H\right)$ behaves as a polynomial in $\log \operatorname{disc}\left(x_{i} H\right)$. We conjecture that this is the only source of bad behavior:

Conjecture 1.11. Fix $\rho>0$. Let $G=\mathbf{G}(\mathbb{R})$ be an $\mathbb{R}$-split real algebraic group, $\Gamma<G$ an arithmetic lattice, and $H$ a maximal $\mathbb{R}$-split torus. Let $x_{i} H$ be a sequence of periodic $H$-orbits satisfying $\operatorname{vol}\left(x_{i} H\right) \geq \operatorname{disc}\left(x_{i} H\right)^{\rho}$. Then any weak limit of the measures $\mu_{x_{i} H}$ is algebraic.

\footnotetext{
${ }^{11}$ More formally, that there is no periodic orbits of a group $L$ with $H<L<G$ containing infinitely many of these periodic $H$-orbits.

${ }^{12}$ Indeed, to prove Theorems 1.4 and 1.6 we use isolation results 5,25 which can be viewed as a poor person's substitute for linearization techniques.
} 
We emphasize that the above Conjecture does not exclude the case of $\mathbf{G}=\mathrm{PGL}_{2}$. At first sight, this may appear too optimistic, in view of the discussion of Section 1.3. However, we expect the large torus orbits considered in Conjecture 1.11 to enjoy a form of rigidity even in the case $\operatorname{dim} H=1$. The reason for this is related to the concept of packets, mentioned in Section 1.2. As remarked there, one may group the compact orbits into collections (packets) which admit an action of an adelic group and have total volume $\operatorname{disc}^{1 / 2+o(1)}$.

It is likely that the large torus orbits considered in Conjecture 1.11 will retain an action of a usable subgroup of this adelic group. This extra action provides a substitute for a rank 2 torus action.

Besides the results of $\S 1.4$, there exists further evidence for Conjecture 1.11, based on recent progress in the analytic theory of $L$-functions. Let us restrict to $\Gamma=\mathrm{PGL}_{n}(\mathbb{Z}), G=\mathrm{PGL}_{n}(\mathbb{R})$. In this context, the exponent $\alpha=1 / 2$ is critical, because the total volume of all orbits of discriminant $D$ is always $\gg_{\epsilon} D^{1 / 2-\epsilon}$. In the case $n=2$, work of the third author with Harcos [17] implies the conjecture for $\alpha$ slightly less than $1 / 2$. Moreover, the GRH implies the conjecture for all $\alpha>1 / 4$. These results use (in addition to, respectively, the results of [17] and the GRH) the relationship between torus orbits and Rankin-Selberg $L$-functions, established in the work of Waldspurger, Katok-Sarnak and Popa (see e.g. [34, Theorem 6.5.1]).

It is worth emphasizing here that $\alpha=1 / 4$ mark the limit of "naive" harmonic analysis. One advantage of the ergodic theoretic methods used in this paper is that results such as Corollary 1.7 yield nontrivial information even for very small $\alpha$. In 14, we shall combine harmonic analysis and ergodic methods to obtain results for $n=3$.

1.7. Analogy with $\times 2 \times 3$. The dynamics of $x \mapsto 2 x, x \mapsto 3 x$ on $\mathbb{R} / \mathbb{Z}$ are, in many ways, similar to the action of the diagonal group on $\mathrm{PGL}_{n}(\mathbb{R}) / \mathrm{PGL}_{n}(\mathbb{Z})$. Here the periodic points are exactly the rationals $x=p / q$ with $(p, q)=1=(q, 6)$.

Then the orbit $2^{n} .3^{m} . x$ has two natural invariants: the size $g$ (i.e. the order of the group $\left.\langle 2,3\rangle \subset(\mathbb{Z} / q \mathbb{Z})^{\times}\right)$and the denominator $q$. These are analogues of the volume and discriminant of a compact $H$-orbit. Again, the relation between these two is a mystery, especially as far as how small $g$ can be; we do not even know that $g \geq 100(\log q)^{2}$.

In their paper [3] Bourgain, Glibichuk and Konyagin prove:

Theorem 1.12 (Bourgain-Glibichuk-Konyagin). Let $\rho>0$ be fixed. Let $q$ be prime and let $G \subset(\mathbb{Z} / q \mathbb{Z})^{\times}$be a subgroup satisfying $|G|>q^{\rho}$. Then for any $a \in(\mathbb{Z} / q \mathbb{Z})^{\times}$, we have:

$$
\left|\sum_{x \in G} e^{2 \pi i a x / q}\right| \leq|G| q^{-\delta}
$$

where $\delta>0$ depends only on $\rho$.

In particular, if $q$ is a prime so that the order of $\langle 2,3\rangle \subset(\mathbb{Z} / q \mathbb{Z})^{\times}$is large, then this theorem implies the periodic orbits of $\times 2 \times 3$ with denominator $q$ are equidistributed individually, as $q \rightarrow \infty$, in analogy with Conjecture 1.11. The restriction that $q$ be prime has been lifted in [4] and subsequent work.

The analogy with $\times 2 \times 3$ is also useful to illustrate, in an explicit context, many of the ideas that are used in this paper. In this vein, we present the analogue of Corollary 1.7 as well as a sketch of its proof. 
Proposition 1.13. Fix $\rho>\rho^{\prime}>0$ and a subinterval $J \subset \mathbb{R} / \mathbb{Z}$. Let $S \subset q^{-1} \mathbb{Z} / \mathbb{Z}$ be invariant under $x \mapsto 2 x, x \mapsto 3 x$ and so that $|S|>q^{\rho}$. Then, for sufficiently large $q$, we have

$$
\frac{|S \cap J|}{|S|} \geq \rho^{\prime} \text { length }(J)
$$

Equivalently: let $S_{i} \subset q_{i}^{-1} \mathbb{Z} / \mathbb{Z}$ with $\left|S_{i}\right|>q_{i}^{\rho}$ be invariant under $x \mapsto 2 x, x \mapsto 3 x$. Let $\mu_{i}$ be the corresponding normalized measure ${ }^{13}$. Then any weak limit of the $\mu_{i}$ dominates $\rho . \mu_{\text {Lebesgue }}$.

Proof. We make free use of the notion of entropy. ${ }^{14}$ Let $\mathcal{P}$ be the partition of $\mathbb{R} / \mathbb{Z}$ into $[0,1 / 2) \cup[1 / 2,1) ;$ let $\mathcal{P}^{(n)}:=\mathcal{P} \vee[2]^{-1} \mathcal{P} \vee \ldots\left[2^{n-1}\right]^{-1} \mathcal{P}$. Here $[m]^{-1} \mathcal{P}$ denotes the partition into $\{x: m x \in[0,1 / 2)\}$ and its complement.

Let $n_{i}$ be minimal so that $2^{n_{i}}>q_{i}$. Then any two elements of $S_{i}$ lie in distinct parts of the partition $\mathcal{P}^{\left(n_{i}\right)}$. From this it follows that $H_{\mu_{i}}\left(\mathcal{P}^{\left(n_{i}\right)}\right)>\log \left(q_{i}\right)$. By the subadditivity of entropy,

$$
H_{\mu_{i}}\left(\mathcal{P}^{\left(n_{i}\right)}\right) \leq H_{\mu_{i}}(\mathcal{P})+H_{\mu_{i}}\left([2]^{-1} \mathcal{P}\right)+\cdots+H_{\mu_{i}}\left(\left[2^{n_{i}-1}\right]^{-1} \mathcal{P}\right)
$$

Because $\mu_{i}$ is invariant under $x \mapsto 2 x$, the summands on the right-hand side are equal. Therefore, $H_{\mu_{i}}(\mathcal{P}) \geq \frac{\log \left|S_{i}\right|}{n_{i}}$. Passing to the limit, we conclude that any weak limit $\mu$ of the $\mu_{i}$ satisfies:

$$
H_{\mu}(\mathcal{P}) \geq \rho \log 2 .
$$

By a simple variation of this argument, one verifies that $H_{\mu}\left(\mathcal{P}^{(k)}\right) \geq k \rho \log 2$. This implies that the entropy of the transformation $x \mapsto 2 x$ w.r.t. $\mu$ is $\geq \rho \log 2$.

We may now apply the following theorem of Rudolph [41]: A probability measure on $\mathbb{R} / \mathbb{Z}$, ergodic and invariant for $\times 2 \times 3$, which has positive entropy w.r.t. $\times 2$, is Lebesgue measure.

To apply it, we decompose $\mu$ into ergodic components with respect to $\times 2 \times 3$. Because any probability measure on $\mathbb{R} / \mathbb{Z}$ has entropy $\leq \log 2$ w.r.t. $\times 2$, it follows that $\mu$ must dominate a measure $\mu^{\prime}$ with total mass $\geq \rho$ and so that almost every ergodic component of $\mu^{\prime}$ has positive entropy w.r.t. $\times 2$. By Rudolph's theorem, $\mu^{\prime}$ dominates $\rho . \mu_{\text {Lebesgue }}$.

The key points in the above proof were: the fact that elements of $S_{i}$ were wellseparated at a small scale (namely $q_{i}^{-1}$ ); the use of subadditivity of entropy; and Rudolph's theorem. The proof of most of the results of this paper, including Corollary 1.7, will use similar ideas in the $\Gamma \backslash G$ context. The well-separated property is established in Proposition 2.3, and we replace the use of Rudolph's theorem with the results of [11].

Acknowledgements. The present paper is part of a project that began on the occasion of the AIM workshop "Emerging applications of measure rigidity" on June 2004 in Palo Alto. It is a pleasure to thank the American Institute of Mathematics, as well as the organizers of the workshop. We would also like to thank Peter Sarnak for his suggestions, comments, and for his constant encouragement during the elaboration of this paper.

\footnotetext{
${ }^{13}$ That is, $\mu_{i}(f)=\frac{1}{S_{i} \mid} \sum_{x \in S_{i}} f(x)$ for a continuous function $f$ on $\mathbb{R} / \mathbb{Z}$.

${ }^{14}$ See (3.6) for the definition and 22] for background. We will use the fact - subadditivity of entropy - that $\left.H_{\mu}(\mathcal{P} \vee \mathcal{Q}) \leq H_{\mu}(\mathcal{P})+H_{\mu}\right)(\mathcal{Q})$ for two partitions $\mathcal{P}, \mathcal{Q}$.
} 
While working on this project the authors visited the following institutions: Princeton University (Ph. M.), the Center of Mathematical Sciences, Zhejiang University (Ph. M. and A. V.), the Institut des Hautes Etudes Scientifiques (Ph. M. and A. V.). The support of these institutions are gratefully acknowledged.

\section{THE DESCRIPTION OF PERIODIC ORBITS}

Let notation be as in Section 1.2.

Our aim in this section is to discuss the parameterization of periodic Cartan orbits and to attach to each orbit an integral invariant, the discriminant. The point is that periodic orbits will be parameterized by rational points on a certain variety (namely, the variety of tori inside $\mathbf{G}$ ) and the discriminant will be the denominator of the associated point.

(1) Two periodic orbits of discriminant $\leq D$ cannot be too close: if $\Omega \subset G$ is compact, and $g_{i} \in \Omega$ are such that $g_{1} H \neq g_{2} H$, and $\Gamma g_{i} H$ are periodic, then the distance between $g_{1}, g_{2}$ is $\gg_{\Omega} D^{-1}$. (See Proposition 2.4, which shows a sharper form of this statement).

(2) A periodic orbit of discriminant $\leq D$ cannot go too close to $\infty$ - this is of relevance only if $\Gamma \backslash G$ is noncompact (Proposition 2.5.)

(3) The volume of a periodic orbit of discriminant $D$ is bounded, up to constants, between $\log D$ and $D^{c}$, for $c>0$ (Proposition 2.8).

(4) The number of periodic Cartan orbits of discriminant $\leq D$ is bounded, up to constants, between $D^{A}$ and $D^{B}$, for $A, B>0$ (Proposition 2.9).

A serious omission in the present account will be that we will avoid any explicit discussion of packets, the natural equivalence relation on periodic orbits. This will be discussed in the paper [14]; see also Remark 1.5 in the present paper.

The paramaterization of periodic orbits that we present here is essentially wellknown; see e.g. [32. The primary goal of this section is, rather, to define the discriminant and show that it crudely controls the dynamics of the orbit, as discussed above.

In Section 4.1 we will specialize some of the discussion presented here to algebraic groups defined by central simple algebras and to the case of $\mathrm{PGL}_{n}$.

2.1. Notation. As a general rule, we will use boldface letters for algebraic varieties, algebraic groups and so forth.

Let $\mathbf{G}$ be a semisimple group over $\mathbb{Q}$ and put $G=\mathbf{G}(\mathbb{R})$. We will assume throughout this document that $\mathbf{G}$ is split over $\mathbb{R}$. While the techniques and ideas extend to the general case, this assumption allows for the most elegant and coherent treatment.

Let $\Gamma$ be an arithmetic subgroup of $G$ that is commensurable with $\mathbf{G}(\mathbb{Q}) \cap$ $\operatorname{GL}(n, \mathbb{Z})$ for some fixed embedding $\rho$ of $\mathbf{G}$ into $\mathrm{GL}(n)$ defined over $\mathbb{Q}$. Let $X=\Gamma \backslash G$ be the associated homogeneous space. Let $\mathfrak{g}=\operatorname{Lie}(\mathbf{G})$ be the Lie algebra of $\mathbf{G}$; thus $\mathfrak{g}$ is a Lie algebra over $\mathbb{Q}$. We fix once and for all a lattice $\mathfrak{g}_{\mathbb{Z}}$ in $\mathfrak{g}$ and a $\mathbf{G}$-invariant nondegenerate bilinear symmetric form $B(\cdot, \cdot)$ defined by

$$
B(X, Y)=\operatorname{Tr}(\rho(X) \rho(Y))
$$

for some fixed faithful representation $\rho$. We assume that these choices have the properties that: $\mathfrak{g}_{\mathbb{Z}}$ is stable by the adjoint action of $\Gamma, B\left(\mathfrak{g}_{\mathbb{Z}}, \mathfrak{g}_{\mathbb{Z}}\right) \subset \mathbb{Z}$, and $\left[\mathfrak{g}_{\mathbb{Z}}, \mathfrak{g}_{\mathbb{Z}}\right] \subset$ $\mathfrak{g}_{\mathbb{Z}}$. We can always find such a $\mathfrak{g}_{\mathbb{Z}}$ (once one has a $\Gamma$-stable lattice, a suitable integral multiple of it will satisfy the latter two properties). 
Let $H$ be an $\mathbb{R}$-split Cartan subgroup of $G$ (i.e. $H$ is the centralizer of a split Cartan subalgebra of $\mathfrak{g} \otimes \mathbb{R}$.) The chosen data fix a Haar measure on $H$ : the form $B$ restricted to the Lie algebra $\mathfrak{h}=\operatorname{Lie}(H)$ is nondegenerate and we shall use the Haar measure on $H$ determined by a top differential form on $\mathfrak{h}$ self-dual with respect to $B$.

Fix an Euclidean norm $\|\cdot\|$ on $\mathfrak{g}$; this also induces an Euclidean norm on $\wedge^{\ell} \mathfrak{g}$ for any $\ell$. The choice of Euclidean norm on $\mathfrak{g}$ determines a left invariant Riemannian metric $d(\cdot, \cdot)$ on $G$ which induces also a metric on $X=\Gamma \backslash G$.

Recall (see, e.g. 46, Remark 3.6]) that for any $R>-1$ the set

$$
\Omega(R)=\left\{\Gamma g:\left\|\operatorname{Ad}\left(g^{-1}\right) v\right\| \geq R^{-1} \text { for all nonzero } v \in \mathfrak{g}_{\mathbb{Z}}\right\}
$$

is compact with $\bigcup_{R} \Omega(R)=\Gamma \backslash G$. It can easily be shown, e.g. using reduction theory ${ }^{15}$ that every $x \in \Omega(R)$ can be represented as $x=\Gamma g$ for some $g$ with $\|\operatorname{Ad}(g)\| \ll R$; here $\|\operatorname{Ad}(g)\|$ denotes the operator norm of $\operatorname{Ad}(g)$. with respect to the fixed Euclidean norm on $\mathfrak{g}$.

Finally, in what follows, we will allow the implicit constant in constructions such as $O(\cdot), \ll, \gg$ to depend on the data fixed above (in particular $\mathbf{G}, \Gamma, \mathfrak{g}_{\mathbb{Z}}$ ).

2.2. The parameterization of periodic orbits. We discuss in this section how to parameterize periodic orbits of $H$ on $X$.

Given a periodic orbit $\Gamma g H$ of $H$ on $X$, the stabilizer in $H$ of any point in this orbit, i.e. $H \cap g^{-1} \Gamma g$, is necessarily a cocompact lattice in $H$. The Zariski closure of $\Gamma \cap g \mathrm{Hg}^{-1}$ is then a torus $\mathbf{T}$ defined over $\mathbb{Q}$ with the property that $\mathbf{T}(\mathbb{R})=g H g^{-1}$. The fact that $\Gamma \cap \mathbf{T}(\mathbb{R})$ is an arithmetic cocompact subgroup of $\mathbf{T}(\mathbb{R})$ assures us that $\mathbf{T}$ is $\mathbb{Q}$-anisotropic. ${ }^{16}$

Proposition 2.1 (Basic correspondence). There is a canonical bijection between

(1) periodic $H$-orbits $\Gamma g H$ on $\Gamma \backslash G$, and

(2) $\Gamma$-orbits on pairs $(\mathbf{T}, g H)$. Here $\mathbf{T}$ is a maximal $\mathbb{Q}$-torus that is anisotropic and $\mathbb{R}$-split, and $g H \in G / H$ is such that $g H^{-1}=\mathbf{T}(\mathbb{R})$, and $\gamma \in \Gamma$ acts via $(\mathbf{T}, g H) \mapsto\left(\gamma \mathbf{T} \gamma^{-1}, \gamma g H\right)$.

Proof. The correspondence has already been indicated: to a periodic $H$-orbit $\Gamma g H$ we associate $(\mathbf{T}, g H)$, where $\mathbf{T}$ is the Zariski closure of $\Gamma \cap g \mathrm{Hg}^{-1}$. In the reverse direction, we associate to $(\mathbf{T}, g H)$ the orbit $\Gamma g H$; it is compact because $\Gamma \cap \mathbf{T}(\mathbb{R})$ is a cocompact lattice in $\mathbf{T}(\mathbb{R})$.

We remark that $\mathbf{T}$, which is necessarily $\mathbb{R}$-split, determines the periodic $H$ orbit up to finitely many possibilities. Indeed, each $\mathbf{T}$ corresponds to precisely one periodic $N_{G}(H)$-orbit, and so to at most $\left|H \backslash N_{G}(H)\right|$ many periodic $H$-orbits.

\footnotetext{
${ }^{15}$ Indeed, fix a maximally $\mathbb{Q}$-split torus $\mathbf{T}_{0} \subset \mathbf{G}$, and let $A_{0}=\mathbf{T}_{0}(\mathbb{R})$. Take $x \in \Omega(R)$. It has by reduction theory a representative $\delta . a . \kappa \in G$, where $a \in A_{0}, \delta$ belongs to a finite subset of $\mathbf{G}(\mathbb{Q})$, and $\kappa$ belongs to a fixed compact subset of $G$. But then $\inf _{v \in \mathfrak{g}_{\mathbb{Z}}}\left\|\operatorname{Ad}(g)^{-1} v\right\|$ is bounded above and below by multiples of $\left\|\operatorname{Ad}\left(a^{-1}\right)\right\|$, because the eigenspaces of $\operatorname{Ad}\left(a^{-1}\right)$ are $\mathbb{Q}$-rational subspaces of $\mathfrak{g}$. Moreover, $\left\|\operatorname{Ad}\left(a^{-1}\right)\right\|$ is bounded above and below by multiples of $\left\|\operatorname{Ad}\left(g^{-1}\right)\right\|$. Finally, note that $\|\operatorname{Ad}(g)\|$ is bounded from above and below by multiples of $\sup _{\|u\|,\|v\|=1}|B(\operatorname{Ad}(g) u, v)|$ and so also by multiples of $\| \operatorname{Ad}\left(g^{-1} \|\right.$.

${ }^{16}$ A character $\chi$ defined on $\mathbf{T}$ over $\mathbb{Q}$ would map the integer points $\mathbf{T}(\mathbb{Z})$ to a subgroup $\chi(\mathbf{T}(\mathbb{Z}))$ of $\mathbb{Q}^{\times}$whose elements allow a common denominator, clearly this subgroup must be trivial and so the same holds for $\chi$ since $\mathbf{T}(\mathbb{Z})$ is a cocompact subgroup of $\mathbf{T}(\mathbb{R})$.
} 
2.3. The discriminant of a periodic orbit. We would like to attach to each periodic orbit $x_{0} H$ a positive integral invariant, the discriminant.

We will do this by means of the correspondence of Proposition 2.1: we will assign a positive integer to each maximal $\mathbb{Q}$-torus contained in $\mathbf{G}$, in a $\Gamma$-invariant fashion. This will be done by interpreting $\mathbb{Q}$-torii as rational points on some variety, and interpreting the discriminant as the "least common denominator" in the coordinates of these points. ${ }^{17}$

Let $r$ be the rank of $\mathbf{G}$. The bilinear form $B$ on $\mathfrak{g}$ gives rise to a bilinear form $B \wedge$ on $\wedge^{r} \mathfrak{g}$ determined by

$$
B_{\wedge}\left(x_{1} \wedge \cdots \wedge x_{r}, y_{1} \wedge \cdots \wedge y_{r}\right)=\operatorname{det}\left(B\left(x_{i}, y_{j}\right)\right)_{i, j=1}^{r} .
$$

Set $V=\wedge^{r} \mathfrak{g} \otimes \wedge^{r} \mathfrak{g}, V_{\mathbb{Z}}=\wedge^{r} \mathfrak{g}_{\mathbb{Z}} \otimes \wedge^{r} \mathfrak{g}_{\mathbb{Z}}$.

For $g \in G$, we will often use the notation $\operatorname{Ad}(g)$ to denote the natural action of $g \in G$ on any of $\mathfrak{g}$ (the adjoint action), $\wedge^{r} \mathfrak{g}$ and $V$.

For any maximal $\mathbb{R}$-split Cartan subalgebra $\mathfrak{t}<\mathfrak{g}$ we define $\iota(\mathfrak{t}) \in V$ by taking any $w \in \wedge^{r} \mathfrak{t}$ and setting

$$
\iota(\mathfrak{t})=\frac{w \otimes w}{B_{\wedge}(w, w)} .
$$

Since the form $B$ restricted to an $\mathbb{R}$-split Cartan subalgebra $t$ is positive definite, for any nonzero $w \in \wedge^{r} \mathfrak{t}$ we have that $B_{\wedge^{r} \mathfrak{g}}(w, w)>0$. If $\mathfrak{t}$ is the Lie algebra of a $\mathbb{Q}$-torus, $\mathfrak{t}_{\mathbb{Z}}=\mathfrak{t} \cap \mathfrak{g}_{\mathbb{Z}}$ is a lattice in $\mathfrak{t}$. Choose a basis $e_{1}, \ldots, e_{r}$ of $\mathfrak{t}_{\mathbb{Z}}$; then $w=e_{1} \wedge \cdots \wedge e_{r}$ is a primitive element of $\wedge^{r} \mathfrak{g}_{\mathbb{Z}}, B_{\wedge}(w, w)$ is a positive integer, and is the smallest integer $n$ such that $n \iota(\mathfrak{t}) \in V_{\mathbb{Z}}$.

The map $g \mapsto g \mathfrak{h} g^{-1}$ gives a bijection between $G / N_{G}(H)$ and $\mathbb{R}$-split Cartan subalgebras of $\mathfrak{g}$, hence we can view $\iota$ as a map from $G / N_{G}(H)$ to $V$. If $\Gamma g H$ is a periodic $H$ orbit $g \mathfrak{h} g^{-1}$ is the Lie algebra of an $\mathbb{R}$-split $\mathbb{Q}$-torus of $G$.

Definition 2.2. Let $\mathfrak{t}$ be the Lie algebra of a maximal $\mathbb{R}$-split $\mathbb{Q}$-torus of $G$; We define the discriminant $\operatorname{disc}(\mathfrak{t})=\min \left\{n \in \mathbf{N}: n \iota(\mathfrak{t}) \in V_{\mathbb{Z}}\right\}$. We define the discriminant of the periodic orbit $\Gamma g H$ to be the discriminant of $g \mathfrak{h} g^{-1}$.

We remark that $\operatorname{disc}(\operatorname{Ad}(\gamma) \mathfrak{t})=\operatorname{disc}(\mathfrak{t})$ for $\gamma \in \Gamma$, by virtue of the assumption that $\mathfrak{g}_{\mathbb{Z}}$ is invariant under the adjoint action of $\Gamma$, and hence the discriminant of a periodic orbit is well-defined.

2.4. Discriminant, discreteness and related properties of closed orbits. The definition of the discriminant of periodic $H$-orbits, and its interpretation in terms of integral points on hypersurfaces, imply several useful facts. The fact that integral points are spaced by $\geq 1$ translates to the following basic proposition, showing that two periodic $H$-orbits of small discriminant cannot be too close. Though simple, this observation is absolutely crucial to our approach.

Proposition 2.3. (Discreteness of periodic orbits - weaker form.) Let $\Omega \subset G$ be a fixed compact subset of $G$. Suppose $g_{1}, g_{2} \in \Omega$ are so that $\Gamma g_{i} H$ are periodic orbits of discriminant $\leq D$ with $g_{1} N_{G}(H) \neq g_{2} N_{G}(H)$. Then $d\left(g_{1}, g_{2}\right) \gg_{\Omega} D^{-2}$.

\footnotetext{
${ }^{17}$ We remark that this "discriminant" is not a canonical construction, e.g. it depends on the choices of the lattice $\mathfrak{g}_{\mathbb{Z}}$ and the bilinear symmetric form $B(\cdot, \cdot)$. However, as we will see later we have set it up so that it agrees with the notion of discriminant for a number field or order (assuming the right choices have been made).
} 
Note that we do not assume that $\Gamma g_{1} N_{G}(H) \neq \Gamma g_{2} N_{G}(H)$, i.e. the proposition also describes how close a particular periodic orbit can come to itself.

Proof. Let $\mathfrak{t}_{i}=\operatorname{Ad}\left(g_{i}\right) \mathfrak{h}$. Then $\left\|\iota\left(\mathfrak{t}_{1}\right)-\iota\left(\mathfrak{t}_{2}\right)\right\| \gg D^{-2}$, as is clear from (2.2). But the map $g \mapsto \iota(\operatorname{Ad}(g) \mathfrak{h})$ is a smooth map from $\Omega$ to $\wedge^{r} \mathfrak{g}$; in particular, it cannot increase distances by more than a constant factor depending on $\Omega$.

Actually we prefer to give a slightly sharper version of this result, which both explicates the dependence on $\Omega$ and gives better dependence on $D$.

Proposition 2.4. (Discreteness of periodic orbits - sharper form.) Suppose $g_{1}, g_{2}$ satisfy $\left\|\operatorname{Ad}\left(g_{i}\right)\right\| \leq R$ and are such that $\Gamma g_{i} H$ are periodic orbits of discriminant $D_{i}$ respectively with $g_{1} N_{G}(H) \neq g_{2} N_{G}(H)$ Then

$$
d\left(g_{1}, g_{2}\right) \gg\left\{\begin{array}{ll}
R^{-r} D^{-1 / 2} & D=D_{1}=D_{2} \\
R^{-2 r}\left(D_{1} D_{2}\right)^{-1 / 2} & D_{1} \neq D_{2}
\end{array} .\right.
$$

Proof. Let $\Gamma g_{i} H$ be periodic, $\mathfrak{t}_{i}=g_{i} \mathfrak{h} g_{i}^{-1}$ and $D_{i}=\operatorname{disc}\left(\Gamma g_{i} H\right)$. Let $w_{0} \in \wedge^{r} \mathfrak{h}$ be such that $B_{\wedge}\left(w_{0}, w_{0}\right)=1$ (this fixes $w_{0}$ up to sign), and set $w_{i}=\operatorname{Ad}\left(g_{i}\right)\left[w_{0}\right]$. Since $g_{1} N_{G}(H) \neq g_{2} N_{G}(H)$ we have that $w_{1} \neq w_{2}$.

It follows from the definition of discriminant that $D_{i}^{1 / 2} w_{i} \in \wedge^{r} \mathfrak{g}_{\mathbb{Z}}$. Indeed, if $e_{1}, \ldots, e_{r}$ are a basis of $\mathfrak{t}_{i} \cap \mathfrak{g}_{\mathbb{Z}}$ then $w=e_{1} \wedge \cdots \wedge e_{r} \in \wedge^{r} \mathfrak{g}_{\mathbb{Z}}$ is an element of $\wedge^{r} \mathfrak{t}$ with $B_{\wedge}(w, w)=D_{i}$. On the other hand $D_{i}^{1 / 2} w_{i}$ has the same property; hence $D_{i}^{1 / 2} w_{i}= \pm w \in \wedge^{r} \mathfrak{g}_{\mathbb{Z}}$.

Consider first the case $D_{1}=D_{2}=D$. Then, since $D^{1 / 2} w_{1}$ and $D^{1 / 2} w_{2}$ are two distinct "integral points", we have $\left\|w_{1}-w_{2}\right\| \gg D^{-\frac{1}{2}}$, i.e. $\| \operatorname{Ad}\left(g_{1}\right) w_{0}-$ $\operatorname{Ad}\left(g_{2}\right) w_{0} \| \gg D^{-1 / 2}$.

The operator norm of $\operatorname{Ad}\left(g_{1}\right)$ acting on $\mathfrak{g}$ is $\leq R$; therefore, the operator norm of $\operatorname{Ad}\left(g_{1}\right)$ acting on $\wedge^{r} \mathfrak{g}$ is $\leq R^{r}$, and we conclude that

$$
\left\|w_{0}-\operatorname{Ad}\left(g_{1}^{-1} g_{2}\right) w_{0}\right\| \gg R^{-r} D^{-1 / 2} \text {. }
$$

This shows that $d\left(g_{1}, g_{2}\right)=d\left(e, g_{1}^{-1} g_{2}\right) \gg R^{-r} D^{-1 / 2}$.

Now consider the case $D_{1} \neq D_{2}$. Then $D_{1}^{1 / 2} w_{1}$ and $D_{2}^{1 / 2} w_{2}$ are two nonproportional integer points, hence they span a parallelogram of area $\gg 1$ and so

$$
\left\|D_{2}^{1 / 2}\left(w_{2}-w_{1}\right)\right\|\left\|D_{1}^{1 / 2} w_{1}\right\| \gg 1
$$

As before, the operator norm of $\operatorname{Ad}\left(g_{1}\right)$ on $\wedge^{r} \mathfrak{g}$ is $\leq R^{r}$, so $\left\|w_{1}\right\|=\left\|\operatorname{Ad}\left(g_{1}\right) w_{0}\right\| \ll$ $R^{r}$ and so from (2.3) we get that

$$
\left\|w_{1}-w_{2}\right\| \gg R^{-r}\left(D_{1} D_{2}\right)^{-1 / 2} .
$$

Proceeding again as in the previous case, we see that $d\left(g_{1}, g_{2}\right) \gg R^{-2 r}\left(D_{1} D_{2}\right)^{-1 / 2}$.

The discriminant also bounds how high a periodic $H$ orbit can penetrate the cusps of $\Gamma \backslash G$. Recall (2.1) for the definition of $\Omega(\cdot)$.

Proposition 2.5. Let $\Gamma g H$ be a periodic $H$-orbit of discriminant $D$. Then $\Gamma g \in$ $\Omega\left(c D^{\operatorname{dim} \mathfrak{g} / 2}\right)$ for some constants $c>0$ (independent of $g$ ). 
Proof. The idea is that $\mathfrak{h} \cap \operatorname{Ad}\left(g^{-1}\right) \mathfrak{g}_{\mathbb{Z}}$ has (by definition of discriminant, and lattice reduction) a basis consisting of vectors that are not too long. From this, we need to finesse that the same is actually true for $\operatorname{Ad}\left(g^{-1}\right) \mathfrak{g}_{\mathbb{Z}}$. But if it contained short vectors, they would generate a nilpotent subalgebra $\mathfrak{n}$ normalized by $\mathfrak{h}$; this would mean that $\operatorname{Ad}(g) \mathfrak{n}$ would be a $\mathbb{Q}$-rational nilpotent algebra normalized by $\mathfrak{t}=$ $\operatorname{Ad}(g) \mathfrak{h}$, a contradiction, for the associated torus is anisotropic.

Let $e_{1}, \ldots, e_{r}$ be a basis for $\mathfrak{t} \cap \mathfrak{g}_{\mathbb{Z}}$. Since $B\left(e_{i}, e_{j}\right) \in \mathbb{Z}$ for every $i, j$ and $\operatorname{det}\left(B\left(e_{i}, e_{j}\right)\right)=D$, lattice reduction shows that we can choose the basis $e_{i}$ so that $B\left(e_{i}, e_{i}\right) \leq c_{0} D$, the constant $c_{0}$ depending only on $r$. Since $e_{i}^{\prime}:=\operatorname{Ad}(g) e_{i} \in \mathfrak{h}$, and on $\mathfrak{h}$ the form $B(\cdot, \cdot)$ is positive definite, this implies that

$$
\left\|e_{i}^{\prime}\right\| \leq c_{1}^{\prime} B\left(e_{i}^{\prime}, e_{i}^{\prime}\right)^{1 / 2} \leq c_{1} D^{1 / 2} \quad \text { for } 1 \leq i \leq r .
$$

Therefore, $\mathfrak{h} \cap \operatorname{Ad}\left(g^{-1}\right) \mathfrak{g}_{\mathbb{Z}}$ has a basis of vectors of length $\leq c_{1} D^{1 / 2}$. We may assume $c_{1}>1$, increasing it if necessary.

On the other hand, there is some $c_{2} \geq 1$ so that

$$
\|[v, w]\| \leq c_{2}\|v\|\|w\| .
$$

For $i \geq 0$, let $\mathfrak{g}_{i}$ be the subspace spanned by $X \in \operatorname{Ad}\left(g^{-1}\right) \mathfrak{g}_{\mathbb{Z}}$ with $\|X\| \leq$ $c_{2}^{-1}\left(c_{2} c_{1} D^{1 / 2}\right)^{-i}$. Then for $i \geq 1$ we have $\left[\mathfrak{h}, \mathfrak{g}_{i}\right] \subset \mathfrak{g}_{i-1} ;$ moreover, for any $i, j \geq 1$ we have $\left[\mathfrak{g}_{i}, \mathfrak{g}_{j}\right] \subset \mathfrak{g}_{i+j}$. So there is $i \leq \operatorname{dim}(\mathfrak{g})$ so that $\mathfrak{g}_{i}=\mathfrak{g}_{i+1}$. Then $\mathfrak{n}:=\operatorname{Ad}(g) \mathfrak{g}_{i}$ is, if nonempty, a $\mathfrak{t}=\operatorname{Ad}(g) \mathfrak{h}$-stable nilpotent subalgebra of $\mathfrak{g}$ defined over $\mathbb{Q}$.

It follows that $\mathfrak{t}$ normalizes the nilpotent Lie algebra $\operatorname{Ad}(g) \mathfrak{n}$, which is defined over $\mathbb{Q}$. But this is a contradiction unless $\mathfrak{n}$ is trivial, for $\mathbf{T}$ is anisotropic. ${ }^{18}$

We conclude that $\operatorname{Ad}\left(g^{-1}\right) \mathfrak{g}_{\mathbb{Z}}$ had no nonzero elements of norm $\leq c_{3} D^{-\operatorname{dim} \mathfrak{g} / 2}$.

The exponent here could be considerably improved. It is easy to see, for instance, that the exponent $\operatorname{dim} \mathfrak{g} / 2$ could be replaced by $1 / 2$ for $\mathbf{G}=\mathrm{PGL}_{2}$ (see also Corollary 4.2). This is indeed sharp:

Example 2.6. Let $G=\mathrm{PGL}_{2}(\mathbb{R}), \Gamma=\mathrm{PGL}_{2}(\mathbb{Z}), H$ the group of diagonal matrices and $d$ a square free positive integer. Then for $g=\left(\begin{array}{cc}1 & 1 \\ \sqrt{d} & -\sqrt{d}\end{array}\right)$ the point $\Gamma g$ is periodic under $H$, of discriminant a constant multiple of $d$ (assuming one makes the obvious choices for $\mathfrak{g}_{\mathbb{Z}}$ etc. $\left.{ }^{19}\right)$ Take $v=\left(\begin{array}{ll}0 & 1 \\ 0 & 0\end{array}\right) \in \mathfrak{g}_{\mathbb{Z}}$. Then

$$
\operatorname{Ad}(g) v=\frac{1}{2}\left(\begin{array}{cc}
1 / \sqrt{d} & 1 / \sqrt{d} \\
-1 / \sqrt{d} & -1 / \sqrt{d}
\end{array}\right)
$$

This implies $g \in \Omega\left(c_{0} d^{1 / 2}\right)$ for a suitable absolute constant $c_{0}$.

We recall the following useful fact about $H$-orbits:

Theorem 2.7 (Tomanov and Weiss [46, Thm 1.3]). There is an $R_{0}$ so that for every $x \in \Gamma \backslash G$ we have that $\Omega\left(R_{0}\right) \cap x H \neq \emptyset$.

\footnotetext{
${ }^{18}$ Over an algebraic closure, $\mathfrak{t} \oplus \mathfrak{n}$ is solvable and so contained in a Borel subalgebra, and so all the roots of $\mathbf{T}$ on $\mathfrak{n}$ are positive in a suitable system. This means that the determinant of the action of $\mathbf{T}$ on $\mathfrak{n}$ cannot be trivial, and so defines a nontrivial character of $\mathbf{T}$.

${ }^{19}$ These (unilluminating) choices are set up for $\mathrm{PGL}_{n}(\mathbb{Z}) \backslash \mathrm{PGL}_{n}(\mathbb{R})$ in Section 4 .
} 
There is a relation between the discriminant and the volume of a periodic $H$ orbit. This connection is explicated in the context of central simple algebras in $\$ 4.1$. Here we give the following general (and less accurate) bounds:

Proposition 2.8. Let $\Gamma g_{0} H$ be periodic $H$-orbit. Then

$$
\log \operatorname{disc}\left(\Gamma g_{0} H\right) \ll \operatorname{vol}\left(\Gamma g_{0} H\right) \ll \operatorname{disc}\left(\Gamma g_{0} H\right)^{c}
$$

for some $c$.

Proof. Let $x_{0}=\Gamma g_{0}$ be a point with $x_{0} H$ a periodic $H$-orbit. By Theorem 2.7, we may assume $g_{0}$ is in some fixed compact subset $\Omega \subset G$. Since $g_{0} \in \Omega$, there are generators $h_{1}, \ldots, h_{r}$ of $g_{0}^{-1} \Gamma g_{0} \cap H$ (possibly up to finite index) so that

$$
\operatorname{vol}\left(x_{0} H\right)=\operatorname{vol}\left(g_{0}^{-1} \Gamma g_{0} \backslash H\right) \gg \max _{i} d\left(e, h_{i}\right) .
$$

It follows that, if one chooses a basis of $\mathfrak{g}$ belonging to the lattice $\mathfrak{g}_{\mathbb{Z}}$, then $\operatorname{Ad}\left(\gamma_{i}\right)=$ $\operatorname{Ad}\left(g_{0} h_{i} g_{0}^{-1}\right)$ is represented (w.r.t. this basis) by an integral matrix all of whose coordinates are $\exp \left(O\left(\operatorname{vol}\left(x_{0} H\right)\right)\right)$.

The Lie algebra $g_{0} \mathfrak{h} g_{0}^{-1}$ is precisely the subspace fixed by the action of all $\gamma_{i}$. Thus there is ${ }^{20}$ a nonzero $w \in \wedge^{r} \mathfrak{g}_{\mathbb{Z}} \cap \wedge^{r} g_{0} \mathfrak{h} g_{0}^{-1}$ with $\|w\| \ll \exp \left(O\left(\operatorname{vol}\left(x_{0} H\right)\right)\right)$. One concludes:

$$
\log \operatorname{disc}\left(x_{0} H\right) \leq \log B_{\wedge^{r} \mathfrak{g}}(w, w) \ll \operatorname{vol}\left(x_{0} H\right) .
$$

This proves the lower bound in (2.4).

To prove the upper bound in (2.4), let $\mathfrak{S} \subset G$ be such that $G=\Gamma \mathfrak{S}$ and $\mathfrak{S}$ has finite Haar measure. An example of such a set is furnished by Siegel domains (see 12, Sec. 4]). Let $\mathfrak{S}(R)=\{g \in \mathfrak{S}: \Gamma g \in \Omega(R)\}$. These Siegel domains have the following additional property which will be useful for us (and is easily verified directly from their definition): for a suitable $C, \beta>0$

$$
\mu_{\text {Haar }}\left(B_{R^{-\beta}}(\mathfrak{S}(R))\right)<C \quad \text { for all } R>0
$$

where $B_{\delta}(S)=\{g \in G: d(g, S)<\delta\}$.

Let $D=\operatorname{disc}\left(x_{0} H\right)$. By Proposition 2.5, the orbit $x_{0} H \subset \Gamma \mathfrak{S}\left(c D^{\operatorname{dim} \mathfrak{g} / 2}\right)$, and so we can write $x_{0} H \subset G$ as a (finite or countable) disjoint union $\bigcup_{i \in J} \Gamma S_{i}$ with each $S_{i} \subset g_{i} H \cap \mathfrak{S}\left(c D^{\operatorname{dim} \mathfrak{g} / 2}\right)$ for some $g_{i} \in G$ lying in distinct cosets of $H$. Let $J^{\prime} \subset J$ be such that $g_{i} N_{G}(H) \neq g_{j} N_{G}(H)$ for every $i, j \in J^{\prime}$ and such that

$$
\sum_{i \in J^{\prime}} \operatorname{vol}\left(S_{i}\right)
$$

is maximal among all possible choices of $J^{\prime}$. Then $\sum_{i \in J^{\prime}} \operatorname{vol}\left(S_{i}\right) \gg \operatorname{vol}\left(x_{0} H\right)$.

By Proposition 2.4, for every pair $i, j \in J^{\prime}$ with $i \neq j$ we have that

$$
B_{\delta}\left(S_{i}\right) \cap B_{\delta}\left(S_{j}\right)=\emptyset \quad \text { for } \delta \leq 2 c_{4} D^{-c_{5}}
$$

\footnotetext{
${ }^{20}$ Given integer matrices in $M_{n}(\mathbb{Z})$, all of whom have all matrix entries $\leq N$, it is easy to see Siegel's lemma - that their common kernel, if nonempty, contains an element of length $N^{c(n)}$, where $c(n)$ depends only on $n$.
} 
$0<c_{4}<1$ and $c_{5} \geq \beta$ being some constants independent of $x_{0}$. Then

$$
\begin{aligned}
1 & \gg \mu_{\text {Haar }}\left(B_{c_{4} D^{-c_{5}}}\left(\mathfrak{S}\left(c D^{\operatorname{dim} \mathfrak{g} / 2}\right)\right)\right) \\
& \geq \sum_{i \in J^{\prime}} \mu_{\text {Haar }}\left(B_{c_{4} D^{-c_{5}}}\left(S_{i}\right)\right) \\
& \gg \sum_{i \in J^{\prime}} D^{-c^{\prime}} \operatorname{vol}\left(S_{i}\right) \gg D^{-c^{\prime}} \operatorname{vol}\left(x_{0} H\right) .
\end{aligned}
$$

Proposition 2.9. Let $N(D)$ be the number of periodic orbits with discriminant less than $D$. Then there exists $c_{1}$, $c_{2}$ such that $D^{c_{1}} \ll N(D) \ll D^{c_{2}}$.

First proof of upper bound. Our proof of the upper bound on the volume of a periodic orbit in Proposition 2.8 applies equally well to a union of periodic orbits of a common discriminant. In other words, the proof of that proposition gives that if $x_{1} H, \ldots, x_{n} H$ are distinct periodic $H$ orbits with $\operatorname{disc}\left(x_{1} H\right)=\cdots=\operatorname{disc}\left(x_{n} H\right)=$ $D$ then

$$
\sum_{i} \operatorname{vol}\left(x_{i} H\right) \ll D^{c}
$$

hence the total volume of all periodic orbits of discriminant $\leq D$ is $\ll D^{c+1}$.

By the lower bound in Proposition 2.8, the volume of a periodic $H$-orbit is $\gg 1,{ }^{21}$ and we conclude that $N(D) \ll D^{c+1}$.

Second proof of upper bound. For any periodic orbit $\Gamma g H$ with discriminant $\leq D$ and associated torus $\mathbf{T}$, the point $\iota(\mathfrak{t}) \in V$ defined by (2.2) satisfies $\iota(\mathfrak{t})=\operatorname{Ad}(g) \iota(\mathfrak{h})$. By Theorem 2.7, $g$ may be taken to belong to a fixed compact subset of $G$; therefore $\iota(\mathfrak{t})$ belongs to a fixed compact subset of $V$ and has (by definition of discriminant) denominator $\leq D$ with respect to the lattice $V_{\mathbb{Z}}$. It follows the number of possibilities for $\iota(\mathfrak{t})$ is $\ll D^{\operatorname{dim} V+1}$.

The lower bound in Proposition 2.9 is closely tied to the notion of packets, as well as the implicit actions of adelic groups, which will be discussed further in [14].

Proof for the lower bound. Let $\Gamma g H$ be a periodic orbit, so that $g H^{-1}=\mathbf{T}(\mathbb{R})$ for an anisotropic torus $\mathbf{T} \subset \mathbf{G}$ as in Proposition 2.1. The existence of one such orbit is established in [35, Thm. 2.13]. Then for $\delta \in \mathbf{G}(\mathbb{Q})$, the orbit $\Gamma \delta g H$ is also periodic; moreover, if $\delta_{1}, \delta_{2}$ define distinct classes in $\Gamma \backslash \mathbf{G}(\mathbb{Q}) / \mathbf{T}(\mathbb{Q})$, the orbits $\Gamma \delta_{1} g H$ and $\Gamma \delta_{2} g H$ are distinct.

It suffices, then, to show that there exist "many" elements in $\Gamma \backslash \mathbf{G}(\mathbb{Q}) / \mathbf{T}(\mathbb{Q})$. To do this, we shall make some use of adelic language; this is natural, because the simplest way of even showing that $\mathbf{G}(\mathbb{Q})$ has "many" elements is the fact that $\mathbf{G}(\mathbb{Q})$ is a lattice in $\mathbf{G}(\mathbb{A})$.

In the following argument, $N_{0}$ will denote a sufficiently large integer. If it is taken sufficiently large (depending only on $\mathbf{G}, \Gamma, \mathbf{T}$ ) then all the statements of the form "for $p \geq N_{0}$ " will be valid. Let $\mathbb{A}_{f}$ be the ring of finite adeles, i.e. $\mathbb{A}_{f}$ consists of the restricted product $\left(x_{p}\right) \in \prod \mathbb{Q}_{p}$ where $x_{p} \in \mathbb{Z}_{p}$ for almost all $p$. Pick an open compact subgroup $K_{f}=\prod_{p} K_{p}$ of $\mathbf{G}\left(\mathbb{A}_{f}\right)$ containing $\Gamma$. It is a theorem [33. Theorem 5.1] that the "class number" $K_{f} \backslash \mathbf{G}\left(\mathbb{A}_{f}\right) / \mathbf{G}(\mathbb{Q})$ is finite. Pick, therefore, a

\footnotetext{
${ }^{21}$ Of course, this can also be seen directly and elementarily.
} 
finite set of representatives $\omega_{1}, \ldots, \omega_{r}$ for $K_{f} \backslash \mathbf{G}\left(\mathbb{A}_{f}\right) / \mathbf{G}(\mathbb{Q})$. We may assume that $\omega_{j} \in K_{p}$ for all $p \geq N_{0}$.

Let $\mathcal{P}$ be a set containing prime numbers larger than $N_{0}$; for each $p \in \mathcal{P}$ suppose we are given $g_{p} \in \mathbf{G}\left(\mathbb{Q}_{p}\right)$ which does not belong to $K_{p} \mathbf{T}\left(\mathbb{Q}_{p}\right)$. For each $p \in \mathcal{P}$, there are $\delta_{p} \in \mathbf{G}(\mathbb{Q}), 1 \leq j(p) \leq r, k_{p} \in K_{f}$ so that

$$
g_{p}=k_{p} \omega_{j(p)} \delta_{p} .
$$

We claim the associated cosets $\Gamma \delta_{p} \mathbf{T}(\mathbb{Q})$ are disjoint. For, if we had an equality $\Gamma \delta_{p} \mathbf{T}(\mathbb{Q})=\Gamma \delta_{q} \mathbf{T}(\mathbb{Q})$, we must in particular, have an equality

$$
K_{f} \omega_{j(p)}^{-1} k_{p}^{-1} g_{p} \mathbf{T}(\mathbb{Q})=K_{f} \omega_{j(q)}^{-1} k_{q}^{-1} g_{q} \mathbf{T}(\mathbb{Q}) .
$$

Looking at the " $p$-component" of this shows that $g_{p} \in K_{p} \mathbf{T}\left(\mathbb{Q}_{p}\right)$, a contradiction.

Now let us produce such a collection $\left\{g_{p}\right\}$. Let $K$ be a field over which $\mathbf{T}$ splits. Thus $\mathbf{G}$ also splits over $K$. Let $\alpha$ be a root of $\mathbf{T}$ and $u_{\alpha}: \mathbb{G}_{a} \rightarrow \mathbf{G}$ the corresponding root subgroup. This morphism of algebraic groups is defined over $K$. If $p \geq N_{0}$ is prime and we pick an embedding $K \hookrightarrow \mathbb{Q}_{p}$ (if one exists), we obtain in an obvious way an embedding $u_{\alpha}: \mathbb{G}_{a} \rightarrow \mathbf{G}$ over $\mathbb{Q}_{p}$. We note that such an embedding exists for a positive density of $p$, by the Chebotarev density theorem.

We claim that - for any such $p$ that is sufficiently large, say $p \geq N_{0}$ - we must have $g_{p}:=u_{\alpha}\left(p^{-1}\right) \notin K_{p} \mathbf{T}\left(\mathbb{Q}_{p}\right)$, and moreover the discriminant of the associated periodic orbit $\Gamma \delta_{p} g H$ is $\leq c_{3} p^{c_{4}}$. These two claims together complete the proof of the lower bound.

The first claim may be deduced as follows. Suppose that $g_{p} \in K_{p} \mathbf{T}\left(\mathbb{Q}_{p}\right)$. Then there exists $t_{p} \in \mathbf{T}\left(\mathbb{Q}_{p}\right)$ so that $u_{\alpha}\left(p^{-1}\right) \cdot t_{p}^{-1} \in K_{p}$. The image of $u_{\alpha}$ defines a closed subvariety of the affine algebraic variety $\mathbf{G} / \mathbf{T}$. This means that there exists a regular, right $\mathbf{T}$-invariant function $f_{\alpha}$ on the algebraic variety $\mathbf{G}$, with the property that it extends the function $u_{\alpha}(x) \mapsto x$. For sufficiently large $p$ we would necessarily have $f_{\alpha}\left(K_{p}\right) \subset \mathbb{Z}_{p}$, contradicting the fact that $u_{\alpha}\left(p^{-1}\right) \cdot t_{p}^{-1} \in K_{p}$.

The second claim follows from the following fact. There is a constant $C>0$ so that, for all $p \geq N_{0}$ and for all $K \hookrightarrow \mathbb{Q}_{p}, \operatorname{Ad}\left(g_{p}\right) \cdot\left(V_{\mathbb{Z}} \otimes \mathbb{Z}_{p}\right) \subset p^{-C}\left(V_{\mathbb{Z}} \otimes \mathbb{Z}_{p}\right)$. Here $V_{\mathbb{Z}}$ is as in the definition of discriminant in Section 2.3. Indeed,

$$
\operatorname{Ad}\left(g_{p}\right)\left(V_{\mathbb{Z}} \otimes \mathbb{Z}_{p}\right)=\operatorname{Ad} \circ u_{\alpha}\left(p^{-1}\right)\left(V_{\mathbb{Z}} \otimes \mathbb{Z}_{p}\right) .
$$

Now Ad $\circ u_{\alpha}$ defines a morphism of algebraic groups from $\mathbb{G}_{a}$ to $\operatorname{GL}(V)$ defined over $K$; choosing a $K$-basis for $V$, the different matrix entries of $\operatorname{Ad} \circ u_{\alpha}(x)$ define a collection of one-variable polynomials in $K[x]$. It would suffice to take for $C$ the largest degree of any one of these polynomials.

Proposition 2.8 and Proposition 2.9 together imply that the number of periodic $H$-orbits of volume $\leq R$ is at $\operatorname{most} \exp (c R)$ for some $c$ (cf. 32 where the finiteness of the number of such orbits is established).

This bound can be improved in the specific examples; see, for example, Corollary 4.2, where it is shown that for $X=\operatorname{PGL}(n, \mathbb{Z}) \backslash \operatorname{PGL}(n, \mathbb{R})$ the number of periodic $H$-orbits of volume $\leq R$ is bounded by a bound of the form $\exp \left(c R^{1 / 2}\right)$ which up to the precise value of $c$ is sharp.

\section{Periodic $H$-ORBits AND POSITIVE ENTROPY}

Our main aim in this section is to prove positive entropy for limit measures arising from periodic $H$-orbits, i.e. Linnik's Principle in Theorem 1.9. Throughout this 
section, let $\mathbf{G}$ be an $\mathbb{R}$-split algebraic group defined over $\mathbb{Q}$, let $\Gamma$ be an arithmetic lattice in $G=\mathbf{G}(\mathbb{R})$ (as always commensurable with $\mathbf{G}(\mathbb{Z})$ ), $H$ be an $\mathbb{R}$-split Cartan subgroup of $G$, and $\mathfrak{h}=$ Lie $H$.

For any $x \in \mathfrak{h}$ there is attached a one parameter subgroup $a(t)=\exp (t x)$ of $H$, and for Haar measure $\mu_{\text {Haar }}$ on $\Gamma \backslash G$ one can easily show that the metric (or Kolmogorov-Sinai) entropy of the flow is given by

$$
h_{\mu_{\mathrm{Haar}}}(a(\cdot))=h_{\mu_{\mathrm{Haar}}}(a(1))=\sum_{\alpha \in \Phi} \max (0, \alpha(x))
$$

where $\Phi$ denotes the set of roots of $G$.

Theorem 3.1. Let $x \in$ Lie $H$ and $a(t)=\exp (t x)$ be a one-parameter subgroup of $H$ as above, and $\rho$ an arbitrary positive real number. Let $Y_{i}=\left\{y_{i, 1} H, \ldots, y_{i, n_{i}} H\right\}$ be a collection of periodic $H$-orbits in $X=\Gamma \backslash G$ and $\Delta_{i} \rightarrow \infty$ satisfying

(1) the discriminants of all $y_{i, j} H$ for $j=1, \ldots, n_{i}$ are at most $\Delta_{i}$

(2) the total volume of all the orbits in $Y_{i}$ is bigger than $\Delta_{i}^{\rho}$.

Let $\mu_{i}$ be the sum of the volume measures on the periodic $H$-orbits $y_{i, j} H$ in $Y_{i}$ $\left(1 \leq j \leq n_{i}\right)$, divided by the total volume of these orbits (so that $\mu_{i}$ is a probability measure). Suppose that

(3) $\mu_{i} \rightarrow \mu$ as $i \rightarrow \infty$ in the weak $k^{*}$ topology for some probability measure $\mu$. Then

$$
h_{\mu}(a(\cdot)) \geq \rho \min _{\alpha \in \Phi}|\alpha(x)| / 2 .
$$

If, instead of (1) above, one assumes

(11) the discriminant of all $y_{i, j} H$ for $j=1, \ldots, n_{i}$ is equal to $\Delta_{i}$ then (3.2) can be improved to

$$
h_{\mu}(a(\cdot)) \geq \rho \min _{\alpha \in \Phi}|\alpha(x)| .
$$

For concreteness, we consider explicitly the case of $G \cong \mathrm{SL}(n, \mathbb{R}), H$ the group of the diagonal matrices, $x \in \mathfrak{h}$ the diagonal matrix with entries $(n-1) / 2,(n-$ $3) / 2, \ldots,-(n-1) / 2$, and $a(t)$ the corresponding one parameter subgroup of $H$. In this case the roots are $\alpha(y)=y_{i}-y_{j}$ where $y \in \mathfrak{h}$ a diagonal matrix with entries $y_{1}, \ldots, y_{n}$, and hence by (3.1) gives $h_{\mu_{\text {Haar }}}(a(\cdot))=\left(\begin{array}{c}n+1 \\ 3\end{array}\right)$. It follows that in the notations of Theorem 3.1 we have

$$
h_{\mu}(a(\cdot)) \geq \begin{cases}\frac{\rho h_{\mu_{\mathrm{Haar}}}(a(\cdot))}{2\left(\begin{array}{c}
n+1 \\
3
\end{array}\right)} & \text { under assumption (11) } \\
\frac{\rho h_{\mu_{\mathrm{Haar}}(a(\cdot))}}{\left(\begin{array}{c}
n+1 \\
3
\end{array}\right)} & \text { under assumption (11) } .\end{cases}
$$

Neither of these bounds seem tight, and indeed in some cases a better bounds can be obtained. In particular, for $\mathbf{G}=\mathrm{PGL}_{2}$ one can prove under assumption $\left(1^{\prime}\right)$ a sharp estimate $h_{\mu} \geq 2 \rho$. Such a bound (which is far from being trivial, even in this very simple context), or more precisely a $p$-adic analog of such a bound, can be deduced from Linnik's "basic lemma" in [26], and a simplified and explicit derivation of this bound will be given in [13].

We wish to draw attention to assumption (3) in Theorem 3.1. If $X=\Gamma \backslash G$ is compact then by passing to a subsequence if necessary this assumption is automatically satisfied. On the other hand, if $\Gamma$ is not a uniform lattice in $G$, whether (3) is 
satisfied or not is a rather interesting issue; e.g. for $\mathbf{G}=\mathrm{PGL}_{n}$ it is closely related to analytic properties of Dedekind $\zeta$-functions.

If one is willing to compromise on the quality of the entropy bound (3.2) or $\left(3.2^{\prime}\right)$, it is possible to relax slightly the assumption that $\mu(X)=1$, assuming only that $\mu(X)>c$ for some explicit $c$ (which for large $n$ will be extremely close to 1 ). We do not give the details as this situation does not seem likely to arise in any natural context.

3.1. Entropy and bounds on measures of tubes. In this and the next subsection we review some well-known facts about entropy in a form which will be convenient for our purposes and deduce Theorem 3.1 from the following proposition. Note that the fact that we are dealing with spaces which are not compact causes minor complication in an otherwise straightforward argument. Moreover, because the space might not be compact it is necessary to assume that the weak* limit is a probability measure.

Let $a(t)$ denote a $\mathbb{R}$-diagonalizable one parameter subgroup of a semisimple group $G=\mathbf{G}(\mathbb{R})$ as above. Fix some open neighborhood of the identity $B \subset G$. For any $s<t \in \mathbb{R}^{+}$, denote

$$
B^{(s, t)}=a(-s) B a(s) \cap a(-t) B a(t) .
$$

Proposition 3.2. Suppose $\mu_{i}$ is a sequence of a $(t)$-invariant probability measures

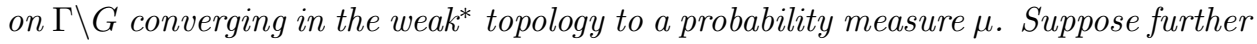
that there is a sequence of positive real numbers $t_{i} \rightarrow \infty$ so that, for every compact $\Omega \subset \Gamma \backslash G$, there exists an open neighborhood of the identity $B \subset G$ with:

$$
\mu_{i} \times \mu_{i}\left(\left\{(x, y) \in \Omega^{2}: y \in x B^{\left(-t_{i}, t_{i}\right)}\right\}\right)<C_{\Omega} e^{-2 \eta t_{i}} .
$$

Then the metric entropy of $\mu$ with respect to the flow a(t) satisfies $h_{\mu}(a(t)) \geq \eta$.

In particular, we have the following:

Corollary 3.3. Let $\mu_{i}, \mu$ be a(t)-invariant probability measures on $\Gamma \backslash G$ with $\mu_{i} \stackrel{w *}{\longrightarrow}$ $\mu$. Suppose that there is a sequence of positive real numbers $t_{i} \rightarrow \infty$ so that, for every compact $\Omega \subset \Gamma \backslash G$, there exists an open neighborhood of the identity $B \subset G$ with

$$
\mu_{i}\left(x B^{\left(-t_{i}, t_{i}\right)}\right)<C_{\Omega}^{\prime} e^{-2 \eta t_{i}} \quad \text { for every } x \in \Omega .
$$

Then the metric entropy of the flow a(t) satisfies $h_{\mu}(a(t)) \geq \eta$.

Proof of Corollary 3.5 assuming Proposition 3.9. By Fubini,

$$
\mu_{i} \times \mu_{i}\left(\left\{(x, y) \in \Omega^{2}: y \in x B^{\left(-t_{i}, t_{i}\right)}\right\}\right)=\int_{\Omega} \mu_{i}\left(x B^{\left(-t_{i}, t_{i}\right)} \cap \Omega\right) d \mu_{i}(x)
$$

applying (3.4) we get

$$
\text { (3.5) } \leq C_{\Omega}^{\prime} \int_{\Omega} e^{-2 \eta t_{i}} d \mu_{i}(x) \leq C_{\Omega}^{\prime} e^{-2 \eta t_{i}}
$$

and hence the condition on $\mu_{i}$ in Corollary 3.3 implies that in Proposition 3.2 .

As we will see below the assumption in Theorem 3.1 regarding the total volume and the information from Proposition 2.4 regarding the discreteness of periodic orbits are enough to deduce the assumptions to Corollary 3.3 . 
Proof of Theorem 3.1 assuming Proposition 3.9. The idea in words is that the assumption (3.4) to Corollary 3.3 expresses an upper bound on the volume of the intersection of collections of periodic orbits with small tubes. However, by Proposition 2.3, pieces of closed orbits can never be too close to each other, so, if the tubes are small enough and the total volume is big enough, a "trivial" bound on the volume of pieces of periodic orbits is sufficient to obtain (3.4) and so positive entropy.

Let $B_{H} \subset H$ be a compact neighborhood of the identity and let $B_{R}=\exp V$ where $V$ is a compact neighborhood of zero in the linear hull of the nonzero root spaces for $\mathfrak{h}$ acting on $\mathfrak{g}$. Then $B=B_{R} B_{H}$ is a neighborhood of the identity $e \in G$. We are going to prove the assumption to Corollary 3.3 for an arbitrary compact set $\Gamma \backslash \Gamma \Omega$ with compact $\Omega \subset G$.

For this, note first that for $t \geq 0$

$$
B^{(-t, t)}=\left(a(t) B_{R} a(-t) \cap a(-t) B_{R} a(t)\right) B_{H} .
$$

Define $\kappa=\min _{\alpha \in \Phi}|\alpha(x)|$. We may assume $a$ is regular and so $\kappa>0$. There exists some constant $c>0$ such that

$$
B^{(-t, t)} \subset B_{c e^{-\kappa t}}^{G}(e) B_{H},
$$

for all $t>0$, where $B_{\delta}^{G}(e)$ denotes a $\delta$-neighbourhood of the identity in $G$.

Recall that if $g_{1}, g_{2} \in \Omega B$ give rise to periodic $H$-orbits of discriminant less than $\Delta_{i}$ then $d\left(g_{1}, g_{2}\right) \gg_{\Omega} \Delta_{i}^{-1}$ unless $g_{1} N_{G}(H)=g_{2} N_{G}(H)$ by Proposition 2.4. Now set $t_{i}=\frac{\log \Delta_{i}}{\kappa}+A$ for some constant $A$ that depends on $B, \Omega$. By choosing $A$ sufficiently large, we can achieve that $g_{1}, g_{2} \in g B^{\left(-t_{i}, t_{i}\right)}$ with $\Gamma g_{1}, \Gamma g_{2} \in Y_{i}$ and $g \in \Omega$ implies $g_{1} N_{G}(H)=g_{2} N_{G}(H)$.

Therefore, $Y_{i} \cap \Gamma g B^{\left(-t_{i}, t_{i}\right)}$ is contained in at most one periodic $N_{G}(H)$-orbit $\Gamma g_{i} N_{G}(H)$ and hence in at most $\left|N_{G}(H) / H\right|$ periodic $H$-orbits. More precisely, there exists $\delta=\delta(\Omega, B)>0$ and $x_{1}, \ldots, x_{J} \in \Gamma g_{i} N_{G}(H)$ with $J=\left|N_{G}(H) / H\right|$ such that

$$
Y_{i} \cap \Gamma g B^{\left(-t_{i}, t_{i}\right)} \subset \bigcup_{j=1}^{J} x_{j} B_{\delta}^{H}(e) .
$$

Since the total volume of $Y_{i}$ with respect to a fixed Haar measure of $H$ is assumed to be $\Delta_{i}^{\rho}$ we see that for the normalized measure

$$
\mu_{i}\left(\Gamma g B^{\left(-t_{i}, t_{i}\right)}\right) \leq \Delta_{i}^{-\rho} \sum_{j} \mu_{H, \text { Haar }}\left(x_{j} B_{\delta}^{H}(e)\right) \ll \Delta_{i}^{-\rho} \ll e^{-\rho \kappa t_{i}} .
$$

This shows (3.4) with $\eta=\frac{\rho \kappa}{2}$ and the theorem in the case of (11). For (1) the proof uses the first case of Proposition 2.4 instead of the second.

3.2. Proof of Proposition 3.2. For any set $\Omega \subset \Gamma \backslash G$, we let

$$
\Omega_{M(\epsilon)}=\left\{x \in \Gamma \backslash G: \sup _{T \in \mathbb{Z}^{+}} \frac{1}{2 T} \sum_{n=-T+1}^{T} 1_{\Omega^{\mathrm{c}}}(x a(-n))<\epsilon\right\}
$$

with $\Omega^{\complement}$ denoting the complement of $\Omega$ in $\Gamma \backslash G$. Note that for $\epsilon<1 / 2$ the above definition particularly implies that $\Omega_{M(\epsilon)} \subset \Omega$. From the maximal inequality, we know that for any $a(t)$-invariant probability measure $\mu$

$$
\mu\left(\Omega_{M(\epsilon)}^{\complement}\right) \leq 10 \epsilon^{-1} \mu\left(\Omega^{\complement}\right) .
$$


For any partition $\mathcal{P}$ of $\Gamma \backslash G$ we let

$$
\mathcal{P}^{(s, t)}=\bigvee_{s \leq n \leq t} \mathcal{P} a(n)
$$

All our partitions will be implicitly assumed to be finite. We will use $[x]_{\mathcal{P}}$ to denote the unique element of $\mathcal{P}$ containing the point $x$. For any finite partition $\mathcal{P}$, we let $H_{\mu}(\mathcal{P})$ denotes its entropy, i.e.

$$
H_{\mu}(\mathcal{P})=-\sum_{P \in \mathcal{P}} \mu(P) \log \mu(P) .
$$

We shall say that the partition $\mathcal{P}$ is $\mu$-regular if for every $P \in \mathcal{P}$ the boundary of $P$ has $\mu$ measure zero.

We now fix a partition $\mathcal{P}$ and a compact set $\Omega \subset \Gamma \backslash G$ which will be used for the remainder of this section. First, we let $\Omega$ be a compact set so that the $\mu$ measure of the interior of $\Omega$ is $>1-\epsilon^{2} / 200$. Then since $\mu_{i} \stackrel{w *}{\longrightarrow} \mu$, for all sufficiently large $i$

$$
\mu_{i}(\Omega)>1-\epsilon^{2} / 100 \text { and } \mu_{i}\left(\Omega_{M(\epsilon)}\right)>1-\epsilon / 10 .
$$

Let $B_{1}$ be a relatively compact symmetric open neighborhood of the identity in $G$ so that:

(1) $B_{1}^{2} \subset B$ with $B$ a neighborhood of the identity satisfying our assumption (3.3)

(2) for any $x \in \Omega$,

$$
x . B_{1} \cap x a(-1) B_{1} a(1)=x B_{1}^{(0,1)} .
$$

We now take $\mathcal{P}$ to be any (finite) $\mu$-regular partition so that for every $x \in \Omega$ the element $[x]_{\mathcal{P}} \subset x B_{1}$ (note that the existence of such partitions is immediate).

Lemma 3.4. Without loss of generality we can choose $B_{1}$ so that for some $C$ and every $n \in \mathbb{Z}^{+}$

$$
\begin{array}{rlrl}
B_{1}^{(0, n)} & \subset F_{n} \cdot B_{1}^{(0, n+1)} & & \text { for some } F_{n} \subset G \text { with }\left|F_{n}\right| \leq C \\
B_{1}^{(-n, 0)} & \subset F_{n} \cdot B_{1}^{(-n-1,0)} & \text { for some } F_{n} \subset G \text { with }\left|F_{n}\right| \leq C .
\end{array}
$$

Proof. We will only prove (3.8); the proof of (3.9) is similar. Let $\mathfrak{g}$ be the Lie algebra of $G$, and let $\mathfrak{g}=\bigoplus_{\lambda} \mathfrak{g}_{\lambda}$ be the decomposition of $\mathfrak{g}$ into eigenspaces for $\operatorname{Ad}(a(t))$. The Riemannian metric defined earlier on $G$ gives us a Euclidean norm on $\mathfrak{g}$. So, for any $\delta$ let $B_{\lambda}(\delta)=\left\{\mathbf{g} \in \mathfrak{g}_{\lambda}:\|\mathbf{g}\|<\delta\right\}$. We take $B_{1}=\exp \left(\sum_{\lambda} B_{\lambda}(\delta)\right)$ where $\delta$ is chosen to be sufficiently small so that $B_{1}^{2} \subset B$ and so that the map exp is a diffeomorphism from $\sum_{\lambda} B_{\lambda}(\delta)$ onto its image. Clearly,

$$
\begin{gathered}
a(t) B_{1} a(-t)=\exp \left(\sum_{\lambda} B_{\lambda}(\exp (t \lambda) \delta)\right) \\
B_{1}^{(s, t)}=\exp \left(\sum_{\lambda \geq 0} B_{\lambda}(\exp (s \lambda) \delta)+\sum_{\lambda<0} B_{\lambda}(\exp (t \lambda) \delta)\right) .
\end{gathered}
$$

Assuming $\delta$ is sufficiently small, for every $\mathbf{g} \in \sum_{\lambda} B_{\lambda}(\delta)$ and $n>0$ we have:

$$
\begin{array}{r}
\exp (\mathbf{g}) \exp \left(\sum_{\lambda \geq 0} B_{\lambda}(\delta / 2) \times \sum_{\lambda<0} B_{\lambda}(\exp ((n+1) \lambda) \delta / 2)\right) \\
\subset \exp \left(\mathrm{g}+\sum_{\lambda \geq 0} B_{\lambda}(\delta) \times \sum_{\lambda<0} B_{\lambda}(\exp ((n+1) \lambda) \delta)\right) ;
\end{array}
$$


This follows easily, e.g. the maps $(X, Y) \mapsto \exp (X+Y)$ and $(X, Y) \mapsto \exp (X) \exp (Y)$ from $\mathfrak{g} \times \mathfrak{g}$ to $G$ have the same derivative at 0 .

Since clearly $\sum_{\lambda \geq 0} B_{\lambda}(\delta) \times \sum_{\lambda<0} B_{\lambda}(\exp (n \lambda) \delta)$ can be covered by a fixed finite number, say $C$, of translates of

$$
\sum_{\lambda \geq 0} B_{\lambda}(\delta / 2) \times \sum_{\lambda<0} B_{\lambda}(\exp ((n+1) \lambda) \delta / 2),
$$

with $C$ independent of $n$, this lemma follows.

Using Lemma 3.4, one easily proves the following using induction: for integers $k \leq 0 \leq m$ and $x \in \Omega$, if

$$
n=\left|\left\{k \leq i \leq m: x a(-i) \in \Omega^{\complement}\right\}\right|
$$

then $[x]_{\mathcal{P}(k, m)} \subset F_{x} \cdot B_{1}^{(k, m)}$ for some $F_{x} \subset \Gamma \backslash G$ satisfying $|F| \leq C^{n}$. In particular, we have the following:

Corollary 3.5. For any $x \in \Omega_{M(\epsilon)}$ and $t \in \mathbb{R}^{+}$there is a finite subset $F_{x, t} \subset \Gamma \backslash G$ with $\log \left|F_{x, t}\right| \ll \epsilon$ so that

$$
[x]_{\mathcal{P}^{(-t, t)}} \subset F_{x, t} \cdot B_{1}^{(-t, t)} .
$$

Lemma 3.6. For every sufficiently large $i$

$$
H_{\mu_{i}}\left(\mathcal{P}^{\left(-t_{i}, t_{i}\right)}\right) \geq \mu_{i}\left(\Omega_{M(\epsilon)}\right)(2 \eta-c \epsilon) t_{i}+O_{\Omega}(1),
$$

with $c$ independent of $\Omega, \epsilon, i$.

Proof. We will first calculate the entropy of $\mathcal{P}^{\left(-t_{i}, t_{i}\right)}$ with respect to the measure $\mu_{i}^{\prime}=\left.\mu_{i}\right|_{\Omega_{M(\epsilon)}}$, i.e. the measure

$$
\mu_{i}^{\prime}(A)=\frac{1}{\mu_{i}\left(\Omega_{M(\epsilon)}\right)} \mu_{i}\left(A \cap \Omega_{M(\epsilon)}\right) .
$$

Let $\left\{P_{1}, \ldots, P_{N}\right\}=\mathcal{P}^{\left(-t_{i}, t_{i}\right)}$, and let $p_{n}^{\prime}=\mu_{i}^{\prime}\left(P_{n}\right)$. By Corollary 3.5, for every $P_{n}$ intersecting $\Omega_{M(\epsilon)}$ (in particular every $P_{n}$ with $p_{n}^{\prime}>0$ ), one has that

$$
P_{n}=\bigcup_{m=1}^{M_{n}} Q_{n m}
$$

with the $Q_{n m}$ disjoint, each $Q_{n m} \subset x_{n m} B_{1}^{\left(-t_{i}, t_{i}\right)}$ for some $x_{n m} \in \Gamma \backslash G$, and $M_{n}<e^{c \epsilon t_{i}}$. Write $q_{n m}^{\prime}=\mu_{i}^{\prime}\left(Q_{n m}\right)$, and let $\mathcal{Q}=\left\{Q_{n m}\right\}$. Then since $\sum_{n} p_{n}^{\prime}=1$, $\sum_{m} q_{n m}^{\prime}=p_{n}^{\prime}$, by convexity of $\log$

$$
H_{\mu_{i}^{\prime}}\left(\mathcal{P}^{\left(-t_{i}, t_{i}\right)}\right)=-\sum_{n} p_{n}^{\prime} \log p_{n}^{\prime} \geq-\log \left(\sum_{i}{p_{n}^{\prime}}^{2}\right)
$$

and finally

$$
\begin{aligned}
\sum_{n}{p_{n}^{\prime}}^{2} & =\sum_{n}\left[\sum_{m} q_{n m}^{\prime}\right]^{2} \leq \sum_{n}\left[\sum_{m} q_{n m}^{\prime}{ }^{2}\right]\left|M_{n}\right| \\
& \leq e^{c \epsilon t_{i}} \sum_{n m} q_{n m}^{\prime}{ }^{2}=e^{c \epsilon t_{i}} \int \mu_{i}^{\prime}\left([x]_{\mathcal{Q}}\right) d \mu_{i}^{\prime}(x) \\
& \leq e^{c \epsilon t_{i}} \int \mu_{i}^{\prime}\left(x B^{\left(-t_{i}, t_{i}\right)}\right) d \mu_{i}^{\prime}(x) \ll \Omega e^{c \epsilon t_{i}-2 \eta t_{i}}
\end{aligned}
$$


by Cauchy-Schwarz, the construction of $\mathcal{Q}$, and the assumption (3.4). It follows that

$$
H_{\mu_{i}^{\prime}}\left(\mathcal{P}^{\left(-t_{i}, t_{i}\right)}\right) \geq(2 \eta-c \epsilon) t_{i}+O_{\Omega}(1)
$$

Let $p_{n}=\mu\left(P_{n}\right)$. Then

$$
\begin{aligned}
H_{\mu_{i}}\left(\mathcal{P}^{\left(-t_{i}, t_{i}\right)}\right) & =-\sum_{n} p_{n} \log p_{n} \geq-\sum_{n} p_{n} \log p_{n}^{\prime} \\
& \geq-\mu_{i}\left(\Omega_{M(\epsilon)}\right) \sum_{n} p_{n}^{\prime} \log p_{n}^{\prime} \\
& =\mu_{i}\left(\Omega_{M(\epsilon)}\right) H_{\mu_{i}^{\prime}}\left(\mathcal{P}^{\left(-t_{i}, t_{i}\right)}\right) \geq \mu_{i}\left(\Omega_{M(\epsilon)}\right)(2 \eta-c \epsilon) t_{i}+O_{\Omega}(1)
\end{aligned}
$$

Proof of Proposition 3.9. By Lemma 3.6, we have, for sufficiently large $i$ :

$$
H_{\mu_{i}}\left(\mathcal{P}^{\left(-t_{i}, t_{i}\right)}\right) \geq \mu_{i}\left(\Omega_{M(\epsilon)}\right)(2 \eta-c \epsilon) t_{i}+O_{\Omega}(1) \geq(1-\epsilon)(2 \eta-c \epsilon) t_{i}
$$

and using subadditivity of $H_{\mu_{i}}$ and invariance of $\mu_{i}$ under $a(t)$ it follows that for any $n$

$$
H_{\mu_{i}}\left(\mathcal{P}^{\left(-t_{i}, t_{i}\right)}\right) \leq \sum_{k=-\left\lfloor t_{i} / n\right\rfloor-1}^{\left\lfloor t_{i} / n\right\rfloor} H_{\mu_{i}}\left(\mathcal{P}^{(-k n,-(k+1) n}\right)=\left(2\left\lfloor t_{i} / n\right\rfloor+2\right) H_{\mu_{i}}\left(\mathcal{P}^{(0, n)}\right) .
$$

Since $\mathcal{P}$ is $\mu$ regular, for any fixed $n$ we have $H_{\mu_{i}}\left(\mathcal{P}^{(0, n)}\right) \rightarrow H_{\mu}\left(\mathcal{P}^{(0, n)}\right)$. Thus

$$
\begin{aligned}
H_{\mu}\left(\mathcal{P}^{(0, n)}\right) & \geq \limsup _{i \rightarrow \infty} \frac{H_{\mu_{i}}\left(\mathcal{P}^{\left(-t_{i}, t_{i}\right)}\right)}{2\left\lfloor t_{i} / n\right\rfloor+2} \\
& \geq(1-\epsilon) \limsup _{i \rightarrow \infty} \frac{2(\eta-c \epsilon) t_{i}}{2\left\lfloor t_{i} / n\right\rfloor+2}=(1-\epsilon)(\eta-c \epsilon) n .
\end{aligned}
$$

Dividing both sides by $n$ and taking the limit as first $n \rightarrow \infty$ and then $\epsilon \rightarrow 0$ gives the proposition.

\section{The action of $H$ on quotients of Central simple algebras}

This section discusses further the case when $\mathbf{G}$ arises from the multiplicative group of an $\mathbb{R}$-split central algebra (e.g., $\mathbf{G}=\mathrm{PGL}_{n}$ !)

In Section 4.1, we explicate the correspondence of Section 2, and discuss (for the only time in this paper) the notion of packet. This notion will be further developed in $[14$.

In Section 4.2, we present and refine some existing theorems about the dynamics of the Cartan action in this setting. In the setting of $\mathbf{G}=\mathrm{PGL}_{3}$ this isolation theorem was proved (in different language) by Cassels and Swinnerton-Dyer.

\subsection{Central simple algebras and $\mathrm{PGL}_{n}$.}

4.1.1. Central simple algebras: notation. Let $D_{\mathbb{Q}}$ be a central simple algebra over $\mathbb{Q}$ of rank $n$, i.e. $D_{\mathbb{Q}} \otimes_{\mathbb{Q}} \overline{\mathbb{Q}}$ is isomorphic to $M_{n}(\overline{\mathbb{Q}})$ as an algebra. We assume that $D_{\mathbb{Q}}$ is split over $\mathbb{R}$, that is to say, $D_{\mathbb{Q}} \otimes_{\mathbb{Q}} \mathbb{R}$ is isomorphic to $M_{n}(\mathbb{R})$.

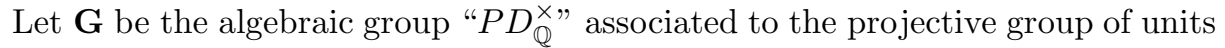
in $D_{\mathbb{Q}}$. The Lie algebra $\mathfrak{g}$ of $\mathbf{G}$ is identified with the quotient of $D_{\mathbb{Q}}$ by its center, the bracket operation being $[x, y]:=x y-y x$.

Let $\mathscr{O}_{D}$ be an order inside $D_{\mathbb{Q}}$, i.e. a $\mathbb{Z}$-module of rank $n^{2}$ containing 1 and closed under multiplication. Choose a basis $\left\{x_{1}, x_{2}, \ldots, x_{n^{2}}\right\}$ for $\mathscr{O}_{D}$; the adjoint 
action of $D_{\mathbb{Q}}$ w.r.t. this basis yields an embedding $\mathbf{G} \rightarrow \mathrm{GL}_{n^{2}}$. The intersection $\mathbf{G}(\mathbb{Q}) \cap \mathrm{GL}_{n^{2}}(\mathbb{Z})$ is commensurable with the image of $\mathscr{O}_{D}^{\times}$in $\mathbf{G}(\mathbb{Q})$; in particular, the image $\Gamma$ of $\mathscr{O}_{D}^{\times}$inside $G=\mathbf{G}(\mathbb{R})$ is an arithmetic lattice. For $\mathfrak{g}_{\mathbb{Z}}$ we take the image of $\mathscr{O}_{D}$ in $\mathfrak{g}$, and finally for $B$ the form

$$
B:(X, Y) \rightarrow \operatorname{tr}(1) \operatorname{tr}(X Y)-\operatorname{tr}(X) \operatorname{tr}(Y),
$$

where $\operatorname{tr}(X)$ is the trace of "left multiplication by $X$ on $D_{\mathbb{Q}}$ ". This defines a bilinear form on $D_{\mathbb{Q}}$ which descends to $\mathfrak{g}$. With these choices it is easy to check that our earlier assumptions hold true.

Furthermore, we fix a maximal commutative totally real semisimple subalgebra $E_{H}$ of $D_{\mathbb{Q}} \otimes \mathbb{R}$; then $E_{H}$ is isomorphic to $\mathbb{R}^{n}$. The centralizer of $E_{H}$ defines a Cartan subgroup $H \subset G$.

4.1.2. Parameterization of closed orbits; lower and upper bounds for volumes. We shall explicate the parameterization of closed orbits in this particular case. Proofs will be given in $\$ 4.1 .3$.

Let $\mathscr{O}$ be a totally real order, i.e. a ring which is a finite free $\mathbb{Z}$-module and so that $\mathscr{O} \otimes \mathbb{Q}$ is a totally real number field. Let $\sigma_{1}, \ldots, \sigma_{n}: \mathscr{O} \otimes \mathbb{Q} \rightarrow \mathbb{R}$ be the different real embeddings. By means of $\theta:=\left(\sigma_{1}, \ldots, \sigma_{n}\right)$ we may regard $\mathscr{O}$ as a lattice in $\mathbb{R}^{n}$; by means of

$$
\eta: x \mapsto\left(\log \left|\sigma_{1}\right|, \ldots, \log \left|\sigma_{n}\right|\right)
$$

we may map $\mathscr{O}^{\times}$(the group of invertible elements in $\mathscr{O}$ ) onto a lattice in $\mathbb{R}^{n-1}=$ $\left\{\left(x_{1}, \ldots, x_{n}\right) \in \mathbb{R}^{n}: \sum_{i} x_{i}\right\}$. The latter result is Dirichlet's unit theorem. We define the discriminant and regulator:

$$
\text { discriminant } \mathscr{O}:=\operatorname{vol}\left(\mathbb{R}^{n} / \theta(\mathscr{O})\right)^{2}, \text { regulator } \mathscr{O}:=\operatorname{vol}\left(\mathbb{R}^{n-1} / \eta\left(\mathscr{O}^{\times}\right)\right)
$$

Proposition 4.1 (Parameterization of periodic orbits for central simple algebras.). In the case of the algebraic group $\mathbf{G}=P D_{\mathbb{Q}}^{\times}$defined by a central simple algebra $D_{\mathbb{Q}}$, we have bijections between:

(1') periodic $H$-orbits on $\Gamma \backslash G$.

(2') $\Gamma$-orbits on pairs $(E, \varphi)$ where $E$ is a subfield of $D_{\mathbb{Q}}$ of degree $n$, and $\varphi$ : $E \otimes \mathbb{R} \rightarrow E_{H}$ is an algebra isomorphism. Here $\gamma$ maps $(E, \varphi)$ to $\left(\gamma E \gamma^{-1}, \varphi \circ\right.$ $\left.\operatorname{Ad}_{D}\left(\gamma^{-1}\right)\right)$. Here $\operatorname{Ad}_{D}\left(\gamma^{-1}\right)$ denotes the conjugation of elements of $D_{\mathbb{Q}}$ by $\gamma^{-1}$.

The bijection associates to the pair $(E, \varphi)$ the periodic orbit $\Gamma g H$, where $g \in G$ has the property that the conjugation $h \rightarrow g h g^{-1}$ maps $E_{H}$ to $E$ and coincides with $\varphi^{-1}$.

The discriminant of the orbit is a constant multiple of the discriminant of the order $E \cap \mathscr{O}_{D}$; the volume of the orbit is a constant multiple of the regulator of $\mathscr{O}_{D}$.

Using this correspondence, we can obtain versions of some of the results from Section 2 with better exponents.

Corollary 4.2. Notations as in the Proposition, we have:

(i) The total volume of all orbits of discriminant $D$ is $\gg_{\epsilon} D^{1 / 2-\varepsilon}$ and the volume of any single periodic orbit of discriminant $D$ satisfies $V \ll_{\epsilon} D^{1 / 2+\varepsilon} .{ }^{22}$

\footnotetext{
${ }^{22}$ This part of the Corollary will not be proved in this paper, but in [14]. However, it is not used anywhere else in the present paper.
} 
(ii) Let $n$ be prime. The volume $V$ of any periodic orbit of discriminant $D$ satisfies $V \gg(\log D)^{n-1}$.

We expect that the exponents of Corollary 4.2 cannot be improved.

To be even more concrete, we specialize further to the case of the split matrix algebra: take $D=M_{n}(\mathbb{Q})$ and $\mathscr{O}_{D}=M_{n}(\mathbb{Z})$, the algebra of $n$ by $n$ matrices. In that case $\mathbf{G}=\mathrm{PGL}_{n}, G=\mathrm{PGL}_{n}(\mathbb{R}), \Gamma=\mathrm{PGL}_{n}(\mathbb{Z})$. Take $E_{H}$ to be the diagonal subalgebra, thus $H$ is the diagonal torus.

Let $K$ be a totally real field of degree $n$.

Definition 4.3. By a lattice in $K$ we shall simply mean a $\mathbb{Z}$-submodule of $K$ of rank $n$. Two lattices $L_{1}, L_{2} \subset K$ are called $K$-equivalent (or simply equivalent) if there is $k \in K^{\times}$so that $k . L_{1}=L_{2}$. Attached to any lattice $L$ we have the associated order $\mathscr{O}_{L}:=\{\lambda \in K: \lambda L \subset L\}$. Then $K$-equivalent lattices have the same associated order.

Corollary 4.4. There is a bijection between:

(1") periodic $H$-orbits on $\mathrm{PGL}_{n}(\mathbb{Z}) \backslash \mathrm{PGL}_{n}(\mathbb{R})$.

(2") Triples $(K, L, \theta)$ of a totally real number field, a $K$-equivalence class of lattices in $K$, and an algebra isomorphism $\theta: K \otimes \mathbb{R} \rightarrow \mathbb{R}^{n}$.

Here the triples of (2") are considered up to isomorphism in the evident sense.

The discriminant of the orbit is a constant multiple of the discriminant of the order $\mathscr{O}_{L}$ associated to $L$, and the volume of the orbit is a constant multiple of the regulator of $\mathscr{O}_{L}$.

If one identifies $\mathrm{PGL}_{n}(\mathbb{Z}) \backslash \mathrm{PGL}_{n}(\mathbb{R})$ with homothety classes of lattices in $\mathbb{R}^{n}$, the bijection assigns to $(K,[L], \theta)$ the $H$-orbit of the homothety class of the lattice $\theta(L)$. Here $L \in[L]$ is arbitrary.

It is often convenient to group the data of $[(2 ")]$ by the order $\mathscr{O}_{L}$. For instance, in the case when $\mathscr{O}_{L}$ is a maximal order, the associated equivalence classes $[L]$ are parameterized by the class group of $\mathscr{O}_{L}$. Thus, approximately speaking, periodic $H$-orbits on $\mathrm{PGL}_{n}(\mathbb{Z}) \backslash \mathrm{PGL}_{n}(\mathbb{R})$ are parameterized by an order in a totally real field, together with an ideal class.

Remark 4.5. There is a natural weaker equivalence relation on lattices $L \subset K$. Namely, we say that $L, L^{\prime}$ are locally equivalent if, for every prime $p$, there exists $\lambda \in\left(K \otimes \mathbb{Q}_{p}\right)^{\times}$so that $\left(L \otimes \mathbb{Q}_{p}\right)=\lambda\left(L^{\prime} \otimes \mathbb{Q}_{p}\right)$. Via the parameterization above, this groups the periodic orbits into finite equivalence classes with the following properties:

(1) Periodic orbits in the same equivalence class have the same volume and same stabilizer in $H$.

(2) Each equivalence class has total volume $V$ satisfying

$$
D^{1 / 2-\epsilon} \ll_{\epsilon} V \ll D^{1 / 2+\epsilon}
$$

where $D$ is the common discriminant.

(3) The set of periodic orbits in each equivalence class is acted on (simply transitively) by a suitable class group.

This type of grouping can be done (after choosing some auxiliary data) in a much more general setting; this is the phenomena of packets that will be discussed further in [14]. 
Again, in this special context we can obtain versions of some results of Section 2 with sharp exponents:

Corollary 4.6. For the $H$-action on $\mathrm{PGL}_{n}(\mathbb{Z}) \backslash \mathrm{PGL}_{n}(\mathbb{R})$ :

(i) There is $c>0$ so that any bounded $H$-orbit of discriminant $D$ is contained in $\Omega\left(c D^{1 / 2}\right)$; the exponent is sharp.

(ii) If $n$ is prime, the number $N(V)$ of periodic $H$-orbits on $\mathrm{PGL}_{n}(\mathbb{Z}) \backslash \mathrm{PGL}_{n}(\mathbb{R})$ of volume $\leq V$ satisfies

$$
V^{1 /(n-1)} \ll \log N(V) \ll V^{1 /(n-1)} .
$$

The behavior of (ii) when $n$ is not prime is heavily influenced by the existence of fields with intermediate subfields. Since the main aim of the corollary is simply to contrast with the case of $n=2$, when one has the asymptotic $\log N(V) \sim V$, we do not attempt to analyze the general case.

4.1.3. Proofs. Let $\mathbf{G}$ be as in Section 4.1.1. Then maximal torii in $\mathbf{G}$ are in bijection with degree $n$ subfields of $D_{\mathbb{Q}}$ : to each maximal torus $\mathbf{T} \subset \mathbf{G}$ one associates the preimage of its Lie algebra, under the natural map $D_{\mathbb{Q}} \rightarrow \mathfrak{g}$.

Proof. (of Prop. 4.1.) The first part of the Proposition is a consequence of Lemma 2.1, taking into account the above remark and the Skolem-Noether theorem [18, Thm. IX.6.7] (which assures that there exists a $g \in G / H$ such that $e \mapsto g^{-1} e g$ coincides with $\left.\varphi: E \otimes \mathbb{R} \rightarrow E_{H}\right)$. For the assertion concerning discriminant, let $\left\{\bar{e}_{1}, \ldots, \bar{e}_{n-1}\right\}$ be a $\mathbb{Z}$-basis for the image of $E \cap \mathscr{O}_{D}$ in the quotient space $E / \mathbb{Q}$; lift them to $e_{1}, \ldots, e_{n-1} \in E \cap \mathscr{O}_{D}$. Then $e_{0}=1, e_{1}, \ldots, e_{n-1}$ is a basis for $E \cap \mathscr{O}_{D}$. The discriminant $D$ of the periodic orbit attached to $(E, g H)$ is

$$
\operatorname{det}\left\{B\left(\bar{e}_{i}, \bar{e}_{j}\right)\right\}=\operatorname{det}\left\{n \operatorname{tr}\left(e_{i} e_{j}\right)-\operatorname{tr}\left(e_{i}\right) \operatorname{tr}\left(e_{j}\right)\right\}_{1 \leq i, j \leq n-1} .
$$

On the other hand,

$\operatorname{disc}\left(E \cap \mathscr{O}_{D}\right)=\operatorname{det}\left\{\operatorname{tr}\left(e_{i} e_{j}\right)\right\}_{0 \leq i, j \leq n-1}=n \operatorname{det}\left\{\operatorname{tr}\left(e_{i} e_{j}\right)-\operatorname{tr}\left(e_{i}\right) \operatorname{tr}\left(e_{j}\right) / n\right\}_{1 \leq i, j \leq n-1}$

From this it follows that $D=n^{n-2} \operatorname{disc}\left(E \cap \mathscr{O}_{D}\right)$.

Proof. (of Corollary 4.2.)

(1) The proof of (i) is most natural after the introduction of packets. We defer it to a later paper. It is not necessary for the proof or statement of any other result in this paper.

(2) For the proof of (ii), the assertion $V \gg(\log D)^{n-1}$, we prove the corresponding fact about orders. If $\mathscr{O}$ is a totally real order of discriminant $D$ and regulator $V($ as in 4.1$)$ ), we will show that $V \gg(\log D)^{n-1}$.

Let $\|\cdot\|$ be the sup norm on $\mathbb{R}^{n}$ defined by $\left\|\left(x_{1}, \ldots, x_{n}\right)\right\|=\max _{i}\left|x_{i}\right|$. We claim that, for any $\lambda \in \mathscr{O}, \lambda \notin \mathbb{Z}$, we have $\|\theta(\lambda)\| \gg D^{\frac{1}{n(n-1)}}$. In fact, $\left\|\theta\left(\lambda^{i}\right)\right\|=\|\theta(\lambda)\|^{i}$ and, because $n$ was prime, $1, \lambda, \ldots, \lambda^{n-1}$ form a $\mathbb{Z}$-basis for the order $\mathbb{Z}[\lambda] \subseteq \mathscr{O}$. Therefore, the covolume of $\mathscr{O}$ is at most $C\|\theta(\lambda)\|^{n(n-1) / 2}$, where $C$ depends only on $n$. Because the covolume of $\mathscr{O}$ is precisely $D^{1 / 2}$, the claim follows.

Therefore, every nonzero vector in $\eta\left(\mathscr{O}^{\times}\right)$has length $\gg_{n} \log D$. Now $\eta\left(\mathscr{O}^{\times}\right)$has rank $n-1$, so it must have covolume $\gg_{n}(\log D)^{n-1}$, as required. 
Proof. (of Corollary 4.4). It follows from Proposition 4.1: periodic $H$-orbits are in bijection with $\Gamma$-orbits on pairs $(K, \theta)$, where $K \subset M_{n}(\mathbb{Q})$ is a totally real field. Given such a pair, we associate to it the triple $(K,[L], \theta)$. Here $L$ is obtained in the following way: fix a nonzero $a \in \mathbb{Q}^{n}$ and let $\jmath(x)=x a$ for $x \in K$. Now set $L=\jmath^{-1}\left(\mathbb{Z}^{n}\right)$.

Conversely, given a triple $(K,[L], \theta)$, choose a basis $\lambda_{1}, \ldots, \lambda_{n}$ for $L$ to obtain an embedding $\iota: K \rightarrow M_{n}(\mathbb{Q})$ with the property that

$$
K \cap M_{n}(\mathbb{Z})=\mathscr{O}_{L}=\{\lambda \in L: \lambda L \subset L\} .
$$

We thereby obtain a pair $(\iota(K), \theta)$ as in the previous section, and moreover the $\Gamma$-orbit of this pair is independent of the choice of basis.

Proof. (of Corollary 4.6) (i) Let $(K,[L], \theta)$ be data parameterizing a periodic orbit; and let $L \subset K$ be any lattice in $K$ in the class $[L]$. If $\alpha \in L$, then also $\alpha \mathscr{O}_{L} \subset L$; here $\mathscr{O}_{L}$ is the order associated to $L$, as in Corollary 4.4. Therefore $\theta(\alpha) \cdot \theta\left(\mathscr{O}_{L}\right) \subset$ $\theta(L)$. Let $D$ be the discriminant of $\mathscr{O}_{L}$, and $\nu$ the volume of $\mathbb{R}^{n} / \theta(L)$. Computing covolumes, this shows for any $\left(x_{1}, \ldots, x_{n}\right) \in \theta(L)$, we have $\prod_{i}\left|x_{i}\right| D^{1 / 2} \geq \nu$.

Writing $\left\|\left(x_{1}, \ldots, x_{n}\right)\right\|=\max _{i}\left|x_{i}\right|$, we can rephrase in the following way: for any $\mathbf{x}=\left(x_{1}, \ldots, x_{n}\right) \in H . \theta(L),\|\mathbf{x}\|^{n} D^{1 / 2} \geq \nu$. It follows from this and lattice reduction that any lattice $\theta\left(L^{\prime}\right) \in H . \theta(L)$ has a reduced basis $v_{1}, \ldots, v_{n}$ with

$$
\nu^{1 / n} D^{-1 /(2 n)} \ll\left\|v_{1}\right\| \ll\left\|v_{2}\right\| \ll \cdots \ll\left\|v_{n}\right\| ; \quad \prod_{i}\left\|v_{i}\right\| \asymp \nu
$$

where the implicit constants in $\ll$ depend only on $n$. But this means also that $\left\|v_{n}\right\| \ll \nu^{1 / n} D^{(n-1) /(2 n)}$.

Now let $\Gamma g H$ belong to the associated periodic orbit. Then $g^{-1} M_{n}(\mathbb{Z}) g$ consists of endomorphisms $X \in M_{n}(\mathbb{R})$ that preserve $\theta\left(L^{\prime}\right)$, for some $L^{\prime} \in H$.L. If $X \in$ $M_{n}(\mathbb{R})$ preserves $\theta\left(L^{\prime}\right)$ and is nonzero, there exists $1 \leq i \leq n$ so that $v_{i} X \neq 0$. But $\left\|v_{i}\right\| \ll \nu^{1 / n} D^{(n-1) /(2 n)}$ and $\left\|v_{i} X\right\| \gg \nu^{1 / n} D^{-1 /(2 n)}$. So the operator norm of $X$ w.r.t. $\|\cdot\|$ is $\gg D^{-1 / 2}$. This implies that $\Gamma g H \in \Omega\left(c D^{1 / 2}\right)$ for suitable $c=c(n)>0$, in the notation of (2.1).

(ii) Let $n$ be prime; we will show that the number $N(V)$ of periodic orbits of volume $\leq V$ satisfies $V^{1 /(n-1)} \ll \log N(V) \ll V^{1 /(n-1)}$.

The upper bound follows from the bound $V \gg(\log D)^{n-1}$, established in Corollary 4.2, as well as Proposition 2.9.

The lower bound follows from Corollary 4.4 and the following fact, also used in Section 7: There exists $a_{n}>0$ and $\gg X^{a_{n}}$ totally real fields $K$ with $\operatorname{disc}(K) \leq X$, each has a maximal order $\mathscr{O}_{K}$ satisfying

$$
\text { regulator }\left(\mathscr{O}_{K}\right) \ll\left(\log \operatorname{discriminant}\left(\mathscr{O}_{K}\right)\right)^{n-1}
$$

and the implicit constants depends only on $n$. See [7, §3, Prop. 1].

4.2. Measure classification and isolation theorems for central simple algebras. Let $G=\mathbf{G}(\mathbb{R})$ be a real algebraic group, $\Gamma<G$ a lattice and $H$ a maximal $\mathbb{R}$-split torus. The dynamics of $H$ is drastically different if $\operatorname{dim} H=1$ or $\operatorname{dim} H>1$, and this is reflected by the behavior of the periodic $H$-orbits.

We study in detail the case $\mathbf{G}=\mathrm{SL}(2)$ in $\$ 7.1$. In this case, and in all cases $\operatorname{dim} H=1$, the behavior of individual periodic $H$-orbits in $\Gamma \backslash G$ is quite arbitrary. When $\operatorname{dim} H \geq 2$ the situation changes dramatically. First consider the case of $\mathrm{PGL}_{n}(\mathbb{Z}) \backslash \mathrm{PGL}_{n}(\mathbb{R})$. Conjecturally, the following is expected to hold: 
Conjecture 4.7. Let $H$ be the diagonal split Cartan subgroup in $\mathrm{PGL}_{n}(\mathbb{R})$ for $n \geq 3$ and consider its right action on $\mathrm{PGL}_{n}(\mathbb{Z}) \backslash \mathrm{PGL}_{n}(\mathbb{R})$.

(1) Every ergodic $H$-invariant probability measure is algebraic, i.e. coincides with the (L-invariant) volume measure of a closed $L$-orbit for some subgroup $H \leq L \leq \mathrm{PGL}_{n}(\mathbb{R})$.

(2) Every bounded $H$-orbit is periodic.

(3) For every compact $\Omega \subset \mathrm{PGL}_{n}(\mathbb{Z}) \backslash \mathrm{PGL}_{n}(\mathbb{R})$ there are only finitely many periodic orbits that are contained in $\Omega$.

Part (11) of this conjecture is a special case of more general conjectures by Furstenberg (unpublished), Katok and Spatzier [20], and Margulis [30], and in particular follows from [30, Conj. 2](properly interpreted); see [12] for more details and more general conjectures).

Part (2) of this conjecture can be traced back to Cassels and Swinnerton-Dyer [5], but in the form given here is due to Margulis (see e.g. [27). It would follow from part (11); this can be shown quite readily using the techniques of [5]. We show below how to deduce part (2) from part (1) using a more refined and recent result.

The same techniques also shows that part (3) of the conjecture follows from part (2). This part of the conjecture has also been highlighted by Margulis (see e.g. [16, Prob. 30]).

One of the important results proved in $[5]$ is an isolation theorem: given any periodic $H$ orbit in $X=\mathrm{PGL}_{n}(\mathbb{Z}) \backslash \mathrm{PGL}_{n}(\mathbb{R})$, and any compact subset $\Omega \subset X$, for any $x \in X$ sufficiently close but not on this periodic $H$ orbit, the orbit $x H$ will intersect the complement of $\Omega$. This has been strengthened by E. L. and B. Weiss 25 for $\mathrm{PGL}_{n}(\mathbb{Z})$ and generalized by $\mathrm{G}$. Tomanov to the split central simple algebra case 45.

Here will need the following slight variant of these results:

Theorem 4.8. Let notation be as in Section 4.1.1, so that $\Gamma$ is a lattice in $G=$ $\mathrm{PGL}_{n}(\mathbb{R})$ associated to a central simple algebra. Let $Y \subseteq X:=\Gamma \backslash G$ be a closed $H$-invariant set. Assume that $Y$ contains a closed orbit $y L$ for a reductive group $L \leq G$ such that

(1) $H \leq L$,

(2) $y L$ has a finite $L$-invariant volume, and

(3) $\overline{Y \backslash(y L)}$ contains $y L$.

Then there exists a strictly bigger reductive $M>L$ such that $y M$ is closed, has finite $M$-invariant volume, and $y M \subseteq Y$. Moreover, if $D_{\mathbb{Q}}=M_{n}(\mathbb{Q})$ so that $\mathbf{G}=\mathrm{PGL}_{n}$, then $Y$ cannot be compact.

The difference between 25, 45] and Theorem 4.8 is that in 25, 45] the set $Y$ is assumed to be of the form $Y=\overline{x H}$ for some $x \in \Gamma \backslash G$ (in which case assumption (3) is automatic unless $Y=y L$ ).

We also remark that, if $n$ is prime, the only possible $L$ as in the statement of the Theorem are $L=H$ and $L=G$. The preimage of the Lie algebra of $L$ in $D_{\mathbb{Q}}$ is actually a semisimple algebra (this statement may be verified over $\mathbb{R}$; now, note that all reductive subgroups of $\mathrm{PGL}_{n}(\mathbb{R})$ that contain a Cartan subgroup are, in fact, Levi subgroups). So the preimage of the Lie algebra of $y L y^{-1}$ in $D_{\mathbb{Q}}$ is a semisimple subalgebra $\mathfrak{l} \subset D_{\mathbb{Q}}$. In order that $y . L$ have finite invariant measure, the group $L$ should not have any $\mathbb{Q}$-characters; this means that $\mathfrak{l}$ must be be a simple $\mathbb{Q}$-algebra; it has dimension $e^{2}$ over its center, some number field $E$. Then $\mathfrak{l} \otimes \mathbb{C}$ is a 
sum of $[E: \mathbb{Q}]$ copies of $M_{e}(\mathbb{C})$; every irreducible representation of it has dimension $e$, so $e \mid n^{2}$. Since $n$ is prime, this forces $e=1$ and $\mathfrak{l}=E$, or $e=n$ and $\mathfrak{l}=D_{\mathbb{Q}}$. In the former case, $L=H$; in the latter case, $L=G$.

Proof of Theorem 4.8. We identify $G \cong \mathrm{PGL}_{n}(\mathbb{R})$ and take $H$ to be the group of diagonal $n \times n$ matrices (with proportional matrices identified). For $a_{1}, \ldots, a_{n}$ we let $\operatorname{diag}\left(a_{1}, \ldots, a_{n}\right)$ denote the diagonal matrix with entries $a_{1}, \ldots, a_{n}$.

The proof of [25, Thm. 1.1] given in [25, pp. 1490-1492] actually proves Theorem 4.8, not just for central simple algebras but for any $\Gamma<\mathrm{PGL}_{n}(\mathbb{R})$, provided the following conditions are satisfied:

- The set $Y$ contains a periodic $H$-orbit $\Gamma g_{0} H$;

- the orbit of $\Gamma g_{0}$ with respect to each of the $\mathbb{R}$-subtori

$$
H_{i j}=\left\{\operatorname{diag}\left(a_{1}, \ldots, a_{n}\right) \in H: a_{i}=a_{j}\right\}
$$

is not closed.

By 35, Thm. 2.13] the orbit $y L$ contains a periodic $H$-orbit $\Gamma g_{0} H$. (In [35] the group is actually assumed to be semisimple. However, $L$ is the product of its center and the semisimple commutator and the same holds up to finite index for its arithmetic lattice. Therefore, the center of $L$ must have compact orbit by the finite volume assumption.)

By Proposition 4.1 and the assumption that $H$ is $\mathbb{R}$-split the periodic orbit $\Gamma g_{0} H$ corresponds to a totally real number field $K \subset D$ of degree $n$. Now consider two indices $1 \leq i<j \leq n$ and the $\mathbb{R}$-subtorus $H_{i j}$ as above. Then $\Gamma \cap g_{0} H_{i j} g_{0}^{-1}=\{\xi \in$ $\left.K \cap \mathscr{O}_{D}^{\times}: \phi(\xi)_{i}=\phi(\xi)_{j}\right\}$ where $\phi: K \rightarrow \mathbb{R}^{n}$ is the algebra homomorphism induced by conjugation by $g_{0}$ as in Lemma 4.1 . Since $\phi(\xi)_{k}$ for $k=1, \ldots, n$ are precisely the various Galois embeddings of $\xi$ into $\mathbb{R}$ and at least two of these give the same value, $\xi$ cannot generate $K$ and so for any $k=1, \ldots, n$ there is a second index $\ell$ with $\phi(\xi)_{k}=\phi(\xi)_{\ell}$. In particular, $\Gamma \cap g_{0} H_{i j} g_{0}^{-1}$ cannot be a lattice in $g_{0} H_{i j} g_{0}^{-1}$ since $n \geq 3$. This establishes the second assumption above.

Using Theorem 4.8 it is easy to prove that (11) implies (2) and (2) implies (3) in Conjecture 4.7.

Indeed,suppose (11) in Conjecture 4.7 holds and let $\mathrm{PGL}_{n}(\mathbb{Z}) g_{0} H$ be a nonperiodic orbit with compact closure $Y$. Then the restriction of the $H$-action on $Y$ has an $H$-invariant and ergodic probability measure $\mu$ which has to be algebraic by our assumption, i.e. $\mu$ is the finite $L$-invariant volume on a closed $L$-orbit for some group $L<G$. Since $H \leq L$ it follows that $L$ is reductive. By Theorem 4.8 it follows that $Y$ cannot be compact - a contradiction.

Suppose (2) holds and there are contrary to (3) infinitely many periodic $H$-orbits within a particular compact set $\Omega$. Let $Y$ be the closure of the union of a sequence of different periodic orbits $\Gamma g_{i} H$ in $\Omega$. Since $\Omega$ is compact there exists a limit point $x$ to a sequence $x_{i} \in \Gamma g_{i} H$. Clearly $x H \subset \Omega$. By our assumption $x$ has itself a compact orbit. Therefore, the assumption to Theorem 4.8 is satisfied with $L=H$ which gives a contradiction to $Y$ being a compact invariant set.

The best known results towards Conjecture 4.7.(1) are under an additional assumption, namely positive entropy. Also note that this conjecture is related to another open question by Furstenberg: What are the probability measures on the circle group $\mathbb{R} / \mathbb{Z}$ invariant under multiplication by 2 and 3 ? The best known result there is Rudolph's theorem [41] saying that an ergodic such measure with positive 
entropy must be the Lebesgue measure. Analogues in higher dimensions and homogeneous spaces were first obtained by Katok and Spatzier [20, 21] under additional assumptions, see also [19]. More recently, two methods were developed [9, 10], [24] for the homogeneous case and together they were sufficient to show that for the $\mathbb{R}$-split Cartan action on $\operatorname{SL}(n, \mathbb{Z}) \backslash \mathrm{SL}(n, \mathbb{R})$ positive entropy for some element is sufficient to deduce that the measure is the volume measure of the homogeneous space [11, Cor. 1.4].

For our purposes, we would like to consider more generally any lattice attached to an order in a central simple algebras as above and not just the special case of $\mathrm{SL}(n, \mathbb{Z})$. This extension (which was suggested to us by Silberman and Tomanov) does not pose additional technical difficulties, and we present it below. Note that examples due to M. Rees [39] show that the theorem below as stated is false for a general lattice $\Gamma$ even if $G=\mathrm{PGL}_{3}(\mathbb{R})$.

Theorem 4.9. Let notation be as in Section 4.1.1, so that $\Gamma$ is a lattice in $G=$ $\mathrm{PGL}_{n}(\mathbb{R})$ associated to a central simple algebra. Suppose $\mu$ is an $H$-invariant and ergodic probability measure on $X=\Gamma \backslash G$ such that the metric entropy $h_{\mu}(a)$ with respect to $\mu$ is positive for some element $a \in H$. Then there exists a reductive $L \leq G$ such that $\mu$ is the $L$-invariant volume form on a single periodic $L$-orbit.

We are following the scheme of proof of [11, Thm. 1.3] which uses as the main tool the more general result 11, Thm. 2.1] about the structure of conditional measures. We refer to [24] or [11, Sec. 2.1] for the basic theory of conditional measures on foliations. We only recall that for every root of $\mathrm{PGL}_{n}(\mathbb{R})$, or more concretely every pair of indices $1 \leq i, j \leq n$ with $i \neq j$, there exists a system of conditional measures $\mu_{x}^{i j}$ for almost every $x \in X$ defined on the corresponding unipotent subgroup $U_{i j}$. Positive entropy means precisely that one such system is nontrivial, i.e. for some pair $i, j$ the conditional measures $\mu_{x}^{i j}$ are not just supported on the identity of $U_{i j}$. If the conditional measures $\mu_{x}^{i j}$ equal the Haar measure of $U_{i j}$ (again for almost every $x$ ), then $\mu$ is in fact invariant under $U_{i j}$. Since the dynamics of the unipotent $U_{i j}$ is much better understood, this situation is desirable. Towards that [11, Thm. 2.1] says that for any $i, j$ one of the following three possibilities take place:

(i) The conditional measures $\mu_{x}^{i j}$ and $\mu_{x}^{j i}$ are trivial a.e.

(ii) The conditional measures $\mu_{x}^{i j}$ and $\mu_{x}^{j i}$ are Haar a.e., and $\mu$ is invariant under left multiplication with elements of $L_{i j}=\left\langle U_{i j}, U_{j i}\right\rangle$.

(iii) Let $A_{i j}^{\prime}=\left\{\operatorname{diag}\left(s_{1}, \ldots, s_{n}\right): \mathbf{s} \in\left(\mathbb{R}^{\times}\right)^{n}\right.$ with $\left.s_{i}=s_{j}\right\}$. Then a.e. ergodic component of $\mu$ with respect to $A_{i j}^{\prime}$ is supported on a single $C\left(L_{i j}\right)$-orbit, where $C\left(L_{i j}\right)=\left\{g: g h=h g\right.$ for all $\left.h \in L_{i j}\right\}$ is the centralizer of $L_{i j}$.

Lemma 4.10. For $X=\Gamma \backslash \mathrm{PGL}_{n}(\mathbb{R})$ as in Theorem 4.9 case (iii) of the above is impossible.

Proof. In [11, Thm. 5.1] it has been shown for $G=\mathrm{SL}_{n}(\mathbb{R})$ that in case (iii) there exists an element $\gamma \in \Gamma$ with the following properties:

(1) diagonalizable over $\mathbb{R}$,

(2) \pm 1 is not an eigenvalue of $\gamma$, and

(3) all eigenvalues of $\gamma$ are simple except precisely one which has multiplicity two.

Note that by rescaling its elements a lattice in $\mathrm{PGL}_{n}(\mathbb{R})$ gives rise to a lattice in $\mathrm{SL}_{n}(\mathbb{R})$. The resulting quotients are isomorphic unless $n$ is even and $\Gamma$ contains 
elements of negative determinant in which case we get a double cover. Because of this we can use the above result also for $G=\mathrm{PGL}_{n}(\mathbb{R})$, for this we define the eigenvalue of $\gamma \in \mathrm{PGL}_{n}(\mathbb{R})$ as the eigenvalue of the matrix after normalizing the determinant to be \pm 1 .

Since $D_{\mathbb{Q}} \otimes \mathbb{R} \cong M_{n}(\mathbb{R})$ the eigenvalues of left multiplication by $\gamma \in \mathscr{O}_{D}^{\times}$on $D_{\mathbb{Q}}$ are precisely the eigenvalues of $\gamma$ when considered as a matrix in $\mathrm{GL}_{n}(\mathbb{R})$, and the multiplicity on $D$ is precisely $n$ times the multiplicity in the matrix. The characteristic polynomial $p(t)$ of left multiplication by $\gamma$ on $D$ therefore factorizes as $p(t)=(t-\xi)^{2 n} \prod_{i=3}^{n}\left(t-\xi_{i}\right)^{n}$ where $\xi, \xi_{3}, \ldots, \xi_{n}$ are the pairwise different eigenvalues of $\gamma$. Since $\gamma \in \mathscr{O}^{\times}$, the polynomial $p(t)$ has integer coefficients and trailing coefficient \pm 1 . If $\xi \notin \mathbb{Q}$, then a Galois conjugate of $\xi$ must be a root of $p(t)$ of the same multiplicity which is impossible by (3). Therefore, $\xi \in \mathbb{Q}$ which forces $\xi= \pm 1$ - a contradiction to (2).

Proof of Theorem 4.9. As explained before our assumption of positive entropy translates to the nontriviality of some conditional measures, i.e. there are pairs of indices $i, j$ for which (i) above fails. By the above lemma (iii) can never hold, so $\mu$ is invariant under $L_{i j}$. Let $L$ be the subgroup generated by all the unipotent subgroups $U_{i j}$ under which $\mu$ is invariant. Clearly $L$ is normalized by $H$, and in fact we can reorder the indices such that $L$ equals $\prod_{i=1}^{\ell} \mathrm{SL}_{m_{i}}(\mathbb{R})$ embedded into $\mathrm{PGL}_{n}(\mathbb{R})$ as block matrices. We will show below that $\sum_{i=1}^{\ell} m_{i}=n$.

By [29, Theorems (a) and (b)], applied to $\mu$ and the group $H L$, we know that there is some $\tilde{L} \geq L$ which is normalized by $H$ so that almost every $L$-ergodic component of $\mu$ is the $\tilde{L}$-invariant measure on a closed $\tilde{L}$ orbit. In particular $\mu$ is $\tilde{L}$-invariant, which unless $\tilde{L} \leq H L$ contradicts the definition of $L$ since otherwise $\tilde{L}$ contains further unipotent subgroups which preserve $\mu$. Let now $x=\Gamma g$ have a closed $\tilde{L}$-orbit $x \tilde{L}$ of finite volume. Then $\Lambda_{\tilde{L}}=g \tilde{L} g^{-1} \cap \Gamma$ is a lattice in $g \tilde{L} g^{-1}$, and so the latter is defined over $\mathbb{Q}$. Therefore, the same is true for the semi-simple $g L g^{-1}=\left[g \tilde{L} g^{-1}, g \tilde{L} g^{-1}\right], \Lambda_{L}=g L g^{-1} \cap \Gamma$ is a lattice in $g L g^{-1}$, and $x L$ is closed with finite volume. However, this implies $\tilde{L}=L$.

Moreover, by [29, Theorems (a) and (b)] $\mu$ is supported by a single orbit of the normalizer $N_{G}(L)$. If in addition $\sum_{i=1}^{\ell} m_{i}=n$, then $N_{G}(L)=H L$ and $\mu$ must be the unique $H L$-invariant measure on the $H L$-orbit. Since $H L$ is reductive, this would prove the theorem.

So suppose $\sum_{i=1}^{\ell} m_{i}<n$. Since $g L g^{-1}$ is a $\mathbb{Q}$-group, so are the normalizer $N_{G}\left(g L g^{-1}\right)$ and its center $Z=C\left(N_{G}\left(g L g^{-1}\right)\right)$. Here $g^{-1} Z g<H$ consists of all diagonal matrices for which all entries in a block corresponding to one of the factors of $L$ are equal to all other entries in the same block, and for which all remaining entries are also equal. Moreover, if $\mathbf{L}^{\prime}, \mathbf{Z}<\mathbf{D}^{\times}$denotes the algebraic group over $\mathbb{Q}$ corresponding to $g L g^{-1}$ and $Z$, then the Lie algebra $\mathfrak{v}$ of $\mathbf{L}^{\prime} \mathbf{Z}$ is a $\mathbb{Q}$-subspace of $D_{\mathbb{Q}}$. Going back to $\mathrm{GL}_{n}(\mathbb{R})$ and the block matrix description of all of the above groups, it is easy to see that $\mathfrak{v}$ is invariant under left multiplication by $\mathbf{Z} \subset \mathbf{D}^{\times}$. The determinant of this representation defines a $\mathbb{Q}$-character of $\mathbf{Z}$. We claim that this determinant is as a character linearly independent from the norm character (defined by the left multiplication on $D_{\mathbb{Q}}$ as a representation). However, this again can be easily seen from the block matrix description: the entries corresponding to the factors of $L$ are used in higher powers than the remaining ones. Therefore, $\mathbf{Z}$ has $\mathbb{Q}$-rank at least two - modulo $\mathfrak{Z}^{\times}$this shows that $Z$ has $\mathbb{Q}$-rank at least 
one. If $h \in g^{-1} Z g$ is such that the value of the $\mathbb{Q}$-character is bigger than one, then it follows that $h^{j} \cdot x \rightarrow \infty$ for $j \rightarrow \infty$ for all points in the $N_{G}(L)$-orbit (where the divergence to infinity is understood within the $N_{G}(L)$-orbit). This contradicts Poincaré recurrence, and so proves the theorem.

\section{DENSITY AND DisTRIBUtion OF PERIODIC ORBITS IN HIGHER RANK}

In this section we employ the tools developed in $\$ 3$ and $\$$ to prove statements about periodic orbits in $\Gamma \backslash G$ with $\Gamma$ a lattice associated to an order in a degree $n$ central simple division algebra, split over $\mathbb{R}$, in $G \cong \mathrm{PGL}_{n}(\mathbb{R})$.

We first use Theorem 3.1 to give a general, if somewhat messy, theorem regarding the distribution of periodic $H$-orbits. Note that if $\Gamma \backslash G$ is noncompact, it is also a conditional result. Later in this section we will deduced from this theorem somewhat cleaner results regarding density properties of these periodic orbits.

Theorem 5.1. Let $\Gamma$ be a lattice in $G \cong \mathrm{PGL}_{n}(\mathbb{R})$ obtained from an order in a central simple algebra of degree $n$, split over $\mathbb{R}$. Let $\rho>0$ be arbitrary. Let $Y_{i}=$ $\left\{y_{i 1} H, \ldots, y_{i n_{i}} H\right\}$ be a collection of periodic $H$-orbits in $X=\Gamma \backslash G$ and $\Delta_{i} \rightarrow \infty$ satisfying

(1) the discriminant of all $y_{i j} H$ is at most $\Delta_{i}$

(2) the total volume of all the orbits in $Y_{i}$ is bigger than $\Delta_{i}^{\rho}$.

Let $\mu_{i}$ be the sum of the volume measures on the compact $H$-orbits $y_{i j} H$ in $Y_{i}$ $\left(1 \leq j \leq n_{i}\right)$, divided by the total volume of these orbits (so that $\mu_{i}$ is a probability measure). Suppose that

(3) $\mu_{i} \rightarrow \mu$ as $i \rightarrow \infty$ in the weak $k^{*}$ topology for some probability measure $\mu$. Then

$$
\mu=\sum_{i=0}^{m} a_{i} \nu_{i}
$$

with $m \in \mathbf{N} \cup\{\infty\}$ and

(a) each $\nu_{i}$ is a $H$-invariant probability measure on $X$, and $a_{i} \geq 0$.

(b) for every $i \geq 1$ the measure $\nu_{i}$ is the $L_{i}$ invariant probability measure on a single periodic orbit of some closed subgroup $L_{i} \leq G$ which properly contains $H$.

(c) $\sum_{i=1}^{m} a_{i} \geq c_{n} \rho$, where $c_{n}$ is a constant which depends only on $n$.

Explicitly, we can take $e^{23}$

$$
c_{n}=\frac{1}{2\left(\begin{array}{c}
n+1 \\
3
\end{array}\right)} .
$$

In particular, $\mu$ is not compactly supported.

Proof. Decompose $\mu$ into its $H$ ergodic components,

$$
\mu=\int \nu d \tau(\nu)
$$

\footnotetext{
${ }^{23}$ If one assumes the discriminant of all $y_{i j} H$ is precisely $\Delta_{i}$, this constant can be improved by a factor of two. In either case this bound is far from optimal - and indeed a better bound can be obtained if one makes a more careful analysis.
} 
where $\tau$ is a probability measure on the space of (Borel) $H$-invariant and ergodic probability measures on $X$. Then, if $a(t)$ is a one-parameter subgroup of $H$,

$$
h_{\mu}(a(\cdot))=\int h_{\nu}(a(\cdot)) d \tau(\nu)
$$

By Theorem 4.9, each such $\nu$ with $h_{\nu}(a(\cdot))>0$ is algebraic: i.e. is the $L$-invariant probability measure on a single periodic $L$ orbit, with $L$ a closed group properly containing $H$ (depending on $\nu$ ), and furthermore each such $\nu$ is not compactly supported. Since there are only finitely many possibilities for $L$, and any such $L$ has only countably many periodic orbits ${ }^{24}$ it follows that we can write $\mu$ as

$$
\mu=a_{0} \nu_{0}+\sum_{i} a_{i} \nu_{i}
$$

with $a_{i} \geq 0, \quad h_{\nu_{0}}(a(\cdot))=0$ and each $\nu_{i}$ an $L_{i}$ invariant probability measure on a single periodic $L_{i}$ orbit, $L_{i}>H$ a closed subgroup of $G$. This establishes (1) and (2) in Theorem 5.1 .

By Theorem 3.1, we know that $h_{\mu}(a(\cdot)) \geq \rho c_{n} h_{\mu_{\text {Haar }}}(a(\cdot))$ for

$$
a(t)=\exp (t \mathbf{h}) \quad \text { with } \mathbf{h}=\operatorname{diag}\left(\frac{n-1}{2}, \frac{n-3}{2}, \ldots, \frac{-n+1}{2}\right) \in \mathfrak{h}
$$

and $c_{n}=\left[2\left(\begin{array}{c}n+1 \\ 3\end{array}\right)\right]^{-1}$. Since, by Ruelle's inequality ${ }^{25}$ the Haar measure on $\Gamma \backslash G$ has maximal entropy, we have:

$$
\begin{aligned}
h_{\mu}(a(\cdot)) & =\int h_{\nu}(a(\cdot)) d \tau(\nu) \\
& =\sum_{i \geq 1} a_{i} h_{\nu_{i}}(a(\cdot)) \leq h_{\mu_{\text {Haar }}}(a(\cdot)) \sum_{i \geq 1} a_{i}
\end{aligned}
$$

it follows that $\sum_{i \geq 1} a_{i} \geq c_{n} \rho$ as claimed.

5.1. Periodic orbits within a fixed compact set. We now turn to proving Theorem 1.4. This theorem states that if $n \geq 3, G=\mathrm{PGL}_{n}(\mathbb{R})$, and $\Gamma=\mathrm{PGL}_{n}(\mathbb{Z})$, for any $\varepsilon>0$, and any compact $\Omega \subset X=\Gamma \backslash G$, the total volume of all periodic $H$ orbits completely contained in $\Omega$ with discriminant $\leq \Delta$ is $\ll_{\varepsilon, \Omega} \Delta^{\varepsilon}$.

Proof of Theorem 1.4. Fix $\Omega, \varepsilon$. Suppose in contradiction that there is some $C$ and an infinite sequence of $\Delta_{i} \rightarrow \infty$ so that, for every $i$, there is a collection of periodic orbits $Y_{i}=\left\{y_{i 1} H, \ldots, y_{i n_{i}} H\right\}$ so that

(i) each $y_{i j} H \subset \Omega$,

(ii) the discriminant $\operatorname{disc}\left(y_{i j} H\right) \leq \Delta_{i}$,

(iii) $\sum_{j=1}^{n_{i}} \operatorname{vol}\left(y_{i j} H\right) \geq C \Delta_{i}^{\varepsilon}$.

\footnotetext{
${ }^{24}$ This countability issue is mostly irrelevant for our purposes, but for completeness suppose $x L$ is periodic for $x=g \Gamma$. Either by [37, Thm 1.1] (where one needs first to show that $x$ is periodic also under $L^{\prime}=[L, L]$ ) or, more directly, by 6, Prop. 2.1] there are only countably many possibilities for $g L g^{-1}$. But $\left\{g^{\prime}: g^{\prime} L g^{\prime-1}=g L g^{-1}\right\}=g N_{G}(L)$ and $\left[N_{G}(L): L\right]<\infty$. Hence given a conjugate $\tilde{L}$ of $L$ there are only finitely many orbits $x L$ in $X$ for which $x$ can be written as $\Gamma g L$ with $g L g^{-1}=\tilde{L}$.

${ }^{25}$ This is usually stated for diffeomorphisms of compact metric spaces. In the context we need, the claim is contained in [28, Theorem 9.7].
} 
Define for each $i$ a probability measure $\mu_{i}$ as in Theorem 5.1. By (i) above, all the measures $\mu_{i}$ are supported on the compact set $\Omega$, and so without loss of generality we can assume that $\mu_{i}$ converge weak ${ }^{*}$ to some probability measure $\mu$ which would also be supported on $\Omega$.

However, $Y_{i}$ satisfy all the assumptions of Theorem 5.1, and it follows that $\mu$ cannot be compactly supported - a contradiction.

5.2. Density of periodic orbits for $\mathbb{R}$-split division algebras. Because (3) of Theorem 5.1 is automatically satisfied, for the compact quotients $X=\Gamma \backslash G$ arising from $\mathbb{R}$-split degree $n \geq 3$ division algebras over $\mathbb{Q}$ one can get more precise information regarding density properties of periodic $H$-orbits. We recall Theorem 1.6 in a slightly more explicit phrasing:

Theorem 1.6. Let $X=\Gamma \backslash G$ be as in (L-2) of the introduction, i.e. $\Gamma$ is a lattice in $G=\mathrm{PGL}_{n}(\mathbb{R})$ associated to a division algebra over $\mathbb{Q}$ (see Section 4.1.1 for details). For any $i$ let $\left(x_{i, j}\right)_{j=1, \ldots, N_{i}}$ be a finite collection of $H$-periodic points with distinct $H$-orbits such that

$$
\sum_{j=1}^{N_{i}} \operatorname{vol}\left(x_{i, j} H\right) \geq C \max _{j}\left(\operatorname{disc}\left(x_{i, j} H\right)\right)^{\rho} .
$$

Suppose that there is no periodic $L$-orbit of a group $H<L<G$ (with both inclusions proper) containing infinitely many $x_{i, j}$. Then $\overline{\bigcup_{i, j} x_{i, j} H}=\Gamma \backslash G$.

Proof. Suppose that the sequence of collections of $H$-periodic orbits

$$
Y_{i}=\left\{y_{i 1} H, \ldots, y_{i j} H\right\} \quad i=1,2, \ldots
$$

forms a counterexample for some fixed $C, \rho$, i.e. this sequence satisfies all the conditions of the above statement, but $\bigcup_{i=1}^{n_{i}} y_{i j} H$ do not become dense. Then there is some open $U \subset X$ so that for every $i, j$ we have that $y_{i j} H \cap U=\emptyset$.

Define probability measures $\mu_{i}$ for each $Y_{i}$ as in the proof of Theorem 1.4, and passing to a subsequence if necessary we may assume that the measures $\mu_{i}$ converge in the weak* topology to a probability measure $\mu$. By Theorem 5.1, $\mu=\sum_{i=0}^{\infty} a_{i} \nu_{i}$ with $a_{i} \geq 0, \quad h_{\nu_{0}}(a(\cdot))=0$ and each $\nu_{i}$ an $L_{i}$ invariant probability measure on a single periodic $L_{i}$ orbit, $L_{i}>H$ a closed subgroup of $G$, and $\sum_{i>1} a_{i} \geq c_{n} \rho$.

Suppose $\nu_{1}$ is a $L_{1}$ invariant probability measure on the periodic orbit $x_{1} L_{1}$, with $a_{1}>0$ and $L_{1}>H$. It follows from [35, Thm. 2.13] (see the beginning of the proof of Theorem 4.8) that $x_{1} L_{1}$ contains a periodic $H$ orbit, say $z H$.

Define

$$
Y=\bigcap_{k \geq 1} \overline{\bigcup_{i \geq k}^{n_{i}} \bigcup_{j=1} y_{i j} H}
$$

Since $U$ is open and disjoint from all $y_{i j} H$, we have that $U \cap Y=\emptyset$. Also, since $\mu_{i}$ converge to $\mu$, and $\mu \geq a_{1} \nu_{1}$, we know that $z L_{1}=x_{1} L_{1} \subset Y$. Let $M \geq L_{1}$ be a maximal closed connected subgroup of $G$ so that the orbit $z M$ is periodic and is contained in $Y$. Clearly $U \cap Y=\emptyset$ implies that $M \neq G$. On the other hand, since $M \neq G$ it follows from the assumptions of Theorem 1.6 that there is some $i_{0}$ so that $y_{i j} \notin z M$ for all $i \geq i_{0}$, hence

$$
Y=\bigcap_{k \geq i_{0}} \overline{\left(\bigcup_{i \geq k} \bigcup_{j=1}^{n_{i}} y_{i j} H\right)} \subset \overline{Y \backslash z M} .
$$


By Theorem 4.8, there is a strictly bigger $\tilde{M}>M$ so that $z \tilde{M}$ is periodic and contained in $Y$ - a contradiction.

\section{Applications to sharpening Minkowski's theorem}

In this Section, we translate one of the foregoing results into number-theoretic terms. As it turns out, it has a rather pleasant application to sharpening an old result of Minkowski.

6.1. Minkowski's theorem. We recall Minkowski's theorem regarding ideal classes, which in particular implies finiteness of the ideal class group:

Theorem 6.1 (Minkowski's theorem). Let $K$ be a number field with maximal order $\mathscr{O}_{K}$. Then any ideal class for $K$ possesses a representative $J \subset \mathscr{O}_{K}$ of norm $N(J)=O(\sqrt{\operatorname{disc}(K)})$ where the implicit constant depends only on $d$.

We conjecture that this is not sharp for totally real number fields of degree $d \geq 3$ insofar as one can replace $O(\cdot)$ by $o(\cdot)$ :

Conjecture 6.2. Suppose $d \geq 3$ is fixed. Then any ideal class in a totally real number fields of degree $d$ has a representative of norm $o(\sqrt{\operatorname{disc}(K)})$.

We expect that this is false for $d=2$. See discussion in Sec. 6.2.

Let $K$ be a number field, $\mathscr{O}_{K}$ its integer ring, and $[J]$ an ideal class of $\mathscr{O}_{K}$. We will denote the regulator of $K$ (more precisely of the integer ring $\mathscr{O}_{K}$ ) by $R_{K}$. Define

$$
\begin{aligned}
m([J], K) & =\min _{J^{\prime} \in[J], J^{\prime} \subset \mathscr{O}_{K}} N(J) \\
m(K) & =\max _{[J]} m([J], K) .
\end{aligned}
$$

where, in the latter definition, the maximum is taken over all ideal classes of $K$. Let $h_{\delta}(K)$ be the number of ideal classes in $K$ with $m([J], K)>\delta \operatorname{disc}(K)^{1 / 2}$.

We prove the following towards Conjecture 6.2.

Theorem 6.3. Let $d \geq 3$, and let $K$ denote a totally real number field of degree $d$. For all $\varepsilon, \delta>0$ we have:

$$
\sum_{\operatorname{disc}(K)<X} R_{K} h_{\delta}(K) \ll_{\varepsilon, \delta} X^{\varepsilon}
$$

In particular:

(1) "Conjecture 6.9 is true for almost all fields": The number of fields $K$ with discriminant $\leq X$ for which $m(K) \geq \delta \cdot \operatorname{disc}(K)^{1 / 2}$ is $O_{\epsilon}\left(X^{\epsilon}\right)$, for any $\epsilon, \delta>0$

(2) "Conjecture 6.2 is true for fields with large regulator": If $K_{i}$ is any sequence of fields for which liminf $\frac{\log R_{K}}{\log \operatorname{disc}(K)}>0$, then $m\left(K_{i}\right)=o\left(\operatorname{disc}\left(K_{i}\right)^{1 / 2}\right)$.

By comparison, we note that Conjecture 6.2 follows from Conjecture 1.3 - see Corollary 6.6. Moreover, if one assumes the GRH one may show that $m(K) \ll_{\varepsilon}$ $(\operatorname{disc} K)^{1+\varepsilon} R_{K}^{-2}$, i.e., one may show a quantitative version of the second assertion of the Theorem, but only in the range when $R_{K}$ is significantly larger than $(\operatorname{disc} K)^{1 / 4}$. The proof of this is very easy, but we postpone it to the paper [14], where we discuss $\zeta$-functions more systematically. 
While the statement of both the Conjecture and Theorem are quite modest (postulating only $o\left(D^{1 / 2}\right)$ instead of $O\left(D^{1 / 2}\right)$ ), we note that Minkowski's bound is in any case very close to sharp:

Proposition 6.4. For any $d \geq 2$ there exists a $c^{\prime}>0$ such that there is an infinite set of totally real fields of degree $d$ for which $m(K) \geq c^{\prime} \cdot \operatorname{disc}(K)^{1 / 2}(\log \operatorname{disc}(K))^{1-2 d}$.

Proof. It has been proved by Duke [8] that there exist infinitely many totally real fields $K$ of degree $d$ whose class number is $\geq c(d) \operatorname{disc}(K)^{1 / 2}(\log \operatorname{disc}(K))^{-d}$, where $c(d)$ is an explicit positive function of $d$. On the other hand, the total number of integral ideals with norm $m$ is bounded by $\sigma_{d}(m)$, where $\sigma_{d}(m)$ is the number of ways of writing $m$ as an ordered product of $d$ non-negative integers. So, the number of integral ideals with norm $\leq X$ is bounded by $\sum_{m \leq X} \sigma_{d}(m) \ll X \log (X)^{d-1}$. Thus there must exist at least one ideal class which has no representative with $\operatorname{norm} \ll \operatorname{disc}(K)^{1 / 2}(\log \operatorname{disc}(K))^{1-2 d}$.

6.2. Translation to dynamics. Throughout this section, let $G=\mathrm{PGL}_{d}(\mathbb{R}), \Gamma=$ $\mathrm{PGL}_{d}(\mathbb{Z}), \quad X=\Gamma \backslash G$, and $H<G$ the group of diagonal matrices.

As we show in Proposition 6.5 below, if $K$ is a totally real number field of a degree $d$ and $\theta: K \otimes \mathbb{R} \rightarrow \mathbb{R}^{d}$ is an algebra isomorphism, $m([J], K)$ is intimately related to how far the $H$-orbit of (the homothety class of) the lattice $\theta\left(J^{-1}\right)$ (considered as an element of $X$ ) penetrates the cusp of $X$. Recall that this orbit is the periodic $H$-orbit associated to the data $\left(K,\left[J^{-1}\right], \theta\right)$, in the notation of Corollary 4.4.

For $d=2$ there is no reason to believe there should be any constraints on such an $H$-orbit, and we expect that Theorem 6.1 cannot be improved in this case. Establishing this rigorously is somewhat complicated because not all periodic $H$ orbits can be obtained as $\theta\left(J^{-1}\right)$ for some ideal $J \subset \mathscr{O}_{K}$ (by Corollary 4.4 the periodic $H$-orbit are encoded by triples $(K,[L], \theta)$, and in this section we are only interested in periodic orbits for which the associated order $\mathscr{O}_{L}$ is the maximal order, i.e. $\left.\mathscr{O}_{K}\right)$.

Because the dynamics of the action of $H$ on $X$ is much more rigid for $d \geq 3$ (see $\S \sqrt{4}$ ), we expect the stronger Conjecture 6.2 to hold, and we show in $\S 6.3$ that Conjecture 6.2 would follow from Conjecture 1.3.

6.3. Small norm representatives of ideal classes and $H$-orbits. For any $\delta>0$ let $\Omega_{\delta}^{\prime} \subset X$ denote the set of homothety classes of lattices $\Lambda<\mathbb{R}^{d}$ containing no vector $v$ with $\|v\|_{\infty}^{d}<\delta \operatorname{covol}(\Lambda)$; equivalently

$$
\Omega_{\delta}^{\prime}=\left\{\Gamma g \in X:\|m g\|_{\infty}^{d} \geq \delta \operatorname{det}(g) \text { for every } m \in \mathbb{Z}^{d}\right\} .
$$

This set is compact by Mahler's Compactness Criterion [36, Cor. 10.9], giving a slightly different system of neighborhoods of infinity to that used earlier (2.1)). We note that $\Omega_{a}^{\prime} \subset \Omega_{b}^{\prime}$ if $a>b$. The previous system of neighbourhoods, however, has the advantage of being defined for a general group.

Proposition 6.5. Let $K$ be a totally real number field of degree $d, \theta_{K}: K \otimes \mathbb{R} \rightarrow \mathbb{R}^{d}$ an algebra isomorphism, and $J$ a fractional ideal of $K$. Let $Y$ be the periodic $H$-orbit on $\Gamma \backslash G$ associated to the data $\left(K,\left[J^{-1}\right], \theta\right)$. Then the following are equivalent:

(a) $m(K,[J])<\delta \operatorname{disc}(K)^{1 / 2}$

(b) $Y$ is not contained in $\Omega_{\delta}^{\prime}$. 
Proof. Let us first notice that, if $\Lambda \subset \mathbb{R}^{d}$ is a lattice, then $H . \Lambda \subset \Omega_{\delta}^{\prime}$ if and only if, for all $\left(x_{1}, \ldots, x_{d}\right) \in L$, we have $\prod_{i}\left|x_{i}\right| \geq \delta \operatorname{covol}(\Lambda)$.

Apply this remark to the lattice $\Lambda=\theta\left(J^{-1}\right)$; recall that $Y$ is precisely the $H$-orbit of this lattice (or, rather, its homothety class). The covolume of $\Lambda$ is $N(J)^{-1}(\operatorname{disc} K)^{1 / 2}$, where $N(J)$ is the norm of the ideal $J$. On the other hand the product of the coordinates of $\theta(x)$ (for $x \in K$ ) is the norm $N(x)$.

Therefore, condition (b) is equivalent to:

$$
\text { There exists } x \in J^{-1}, \quad|N(x)| \leq \delta N(J)^{-1}(\operatorname{disc} K)^{1 / 2} .
$$

But elements $x \in J^{-1}$ map surjectively onto the set of ideals $I \subset \mathscr{O}_{K}$ which belong to the same ideal class as $J$; the map is $x \mapsto x . J$. Therefore, (6.2) translates to condition (a).

Note that, for $\delta$ sufficiently large, $\Omega_{\delta}^{\prime}$ is empty. Thus the Proposition immediately implies Theorem 6.1 (at least for totally real $K$ ). Another direct corollary of Proposition 6.5 is the following:

Corollary 6.6. Conjecture 1.3 implies Conjecture 6.9.

Proof. If Conjecture 6.2 were false, then for some $\delta>0$ there is an infinite sequence of totally real fields $K_{i}$ (equipped with an algebra isomorphism $\theta_{i}: K_{i} \otimes \mathbb{R} \rightarrow \mathbb{R}^{d}$ ) and ideals $J_{i} \subset \mathscr{O}_{K_{i}}$ with $m\left(\left[J_{i}\right], K_{i}\right) \geq \delta \sqrt{\operatorname{disc}(K)}$. By Proposition 6.5 this gives us an infinite sequence of periodic $H$-orbits all inside the fixed compact set $\Omega_{\delta}^{\prime}$, in contradiction to Conjecture 1.3 .

Substituting Theorem 1.4 for Conjecture 1.3, a similar argument gives the following:

Proof of Theorem 6.3. Suppose Theorem 6.3 was false. Then there will be $C, \varepsilon, \delta>$ 0 and a sequence of integers $D_{i} \rightarrow \infty$ so that

$$
\sum_{\operatorname{disc}(K)<D_{i}} R_{K} h_{\delta}(K)>C D_{i}^{\varepsilon} \quad \text { for all } i,
$$

the summation being on totally real number fields of degree $d$. Fix for every totally real field $K$ for which $m(K) \geq \delta \sqrt{\operatorname{disc}(K)}$ an algebra isomorphism $\theta_{K}: K \otimes \mathbb{R} \rightarrow$ $\mathbb{R}^{d}$. Let $\left[J_{K, j}\right]\left(j=1, \ldots, h_{\delta}(K)\right)$ be the ideal classes of $K$ with $m\left(\left[J_{K, j}\right], K\right) \geq$ $\delta \sqrt{\operatorname{disc}(K)}$.

Let $Y_{K, j}$ be the periodic $H$-orbit parameterized (in the language of Corollary 4.4) by $\left(K,\left[J_{K, j}^{-1}\right], \theta_{K}\right)$, i.e. the $H$-orbit of the homothety class of $\theta_{K}\left(J_{K, j}^{-1}\right)$. Corollary 4.4 states that $Y_{K, j}$ are periodic $H$-orbits with volume proportional to $R_{K}$ and discriminant proportional to $\operatorname{disc}(K)$; Proposition 6.5 shows that $Y_{K, j} \subset \Omega_{\delta}^{\prime}$.

Our assumption (6.3) implies that the collections of periodic $H$-orbits

$$
\mathcal{C}_{i}=\left\{Y_{K, j}: K \text { totally real with } \operatorname{disc}(K)<D_{i}, 1 \leq j \leq h_{\delta}(K)\right\} \subset \Omega_{\delta}^{\prime}
$$

have discriminant $\leq D_{i}$ and total volume $\gg D_{i}^{\varepsilon}$, contradicting Theorem 1.4.

\section{EXAMPLES OF PERIODIC ORBITS WHICH DO NOT EQUIDISTRIBUTE}

In this section we discuss in detail the example of $n=2$ (where rigidity for the action of the diagonal group completely breaks down) as well as examples for $n \geq 3$ showing that individual orbits can escape to the cusp. The latter class of examples 
are particularly relevant, because they suggest strongly that some of the hypotheses in our previous theorems cannot be easily removed.

7.1. Abundance of periodic orbits in compact sets in $\mathrm{PGL}_{2}(\mathbb{Z}) \backslash \mathrm{PGL}_{2}(\mathbb{R})$. As we discussed in section 2 there are two natural parameters attached to each periodic $H$-orbit $\Gamma g H$ of an arithmetic quotients $\Gamma \backslash G$. The discriminant $\operatorname{disc}(\Gamma g H)$ which measures the arithmetic complexity of the orbit and the volume (or regulator) of the orbit $\operatorname{vol}(\Gamma g H)$ which is the covolume of the lattice $g^{-1} \Gamma g \cap H$ in $H$. As we have seen it is natural for us to order the periodic orbits by their discriminant but to use the volume as weights when counting the orbits. In this sense the next theorem shows that quite many periodic orbits under the geodesic flow on $\mathrm{PGL}_{2}(\mathbb{Z}) \backslash \mathrm{PGL}_{2}(\mathbb{R})$ belong to a fixed compact set.

Theorem 7.1. Let $G=\mathrm{PGL}_{2}(\mathbb{R}), \Gamma=\mathrm{PGL}_{2}(\mathbb{Z})$, and $X_{2}=\Gamma \backslash G$. Let

$$
H=\left\{\left(\begin{array}{cc}
e^{t / 2} & \\
& e^{-t / 2}
\end{array}\right): t \in \mathbb{R}\right\}
$$

be the diagonal Cartan so that the action of $\mathrm{H}$ on $\mathrm{X}_{2}$ is precisely the geodesic flow on the (unit tangent bundle of the) unimodular surface. Then for every $\epsilon>0$ there exists a $\delta>0$ such that

$$
\sum_{\operatorname{disc}(\Gamma g H) \leq \Delta, \Gamma g H \subset \Omega_{\delta}^{\prime}} \operatorname{vol}(\Gamma g H) \gg \Delta^{1-\epsilon},
$$

where $\Omega_{\delta}^{\prime}$ is the compact set defined in Section 6.S.

In comparison, we remark that without the restriction on the compact set (cf. 44)

$$
\sum_{\operatorname{disc}(\Gamma g H) \leq \Delta} \operatorname{vol}(\Gamma g H) \ll_{\varepsilon} \Delta^{3 / 2+\varepsilon} .
$$

This theorem is just a special case of the general philosophy that the dynamics of $\mathbb{R}$-rank one Cartan groups $H \subset \mathrm{PGL}_{2}$ is very flexible. Using similar methods the theorem can be generalized to periodic orbits arising e.g. from cubic number fields with one real and one complex embedding (where $\mathbb{S}^{1} \times \mathbb{R}^{>0} \cong H<\mathrm{PGL}_{3}(\mathbb{R})$ ). We restrict our attention to the special case of the geodesic flow only for brevity, and to be able to give a simple self contained treatment. We will indicate references for the general case. However, as we have seen the higher rank case is very different, see Section 5 .

For the proof of Theorem 7.1 we will need several lemmata.

Lemma 7.2. (cf. Proposition 2.8) There exists a constant $C>0$ such that

$$
\operatorname{vol}(\Gamma g H) \geq \log \operatorname{disc}(\Gamma g H)-C
$$

for any periodic orbits $\Gamma g H$.

Proof. Since both the volume (i.e. regulator) and the discriminant only depend on the order and not on the proper ideal associated to the orbit in Corollary 4.4, we can assume without loss of generality (replacing, in the notation of that Corollary, " $L$ " by " $\mathscr{O}_{L}$ ") that the orbit is defined by data $(\mathbb{Q}(\sqrt{D}), \mathscr{O}, \theta)$, where $\mathscr{O} \subset \mathbb{Q}(\sqrt{D})$ is the order of discriminant $D$.

By definition $\mathscr{O}$ gives rise to a lattice $\Lambda_{\mathscr{O}}:=\theta(\mathscr{O}) \subset \mathbb{R}^{2}$ of covolume $D^{1 / 2}$. Since $1 \in \mathscr{O}$ we have $(1,1) \in \Lambda_{\mathscr{O}}$ which is a "short vector" in comparison to the covolume. To see better why $(1,1)$ is short, let us renormalize $\Lambda_{\mathscr{O}}$ by a homothety to covolume 
one. Then $\left(D^{-1 / 4}, D^{-1 / 4}\right)$ belongs to the so obtained lattice $\Lambda_{\mathscr{O}}^{1}$. Applying the geodesic flow $h_{t}=\left(\begin{array}{cc}e^{t / 2} & \\ & e^{-t / 2}\end{array}\right)$ (in either direction) makes this vector longer. However, as long as $|t / 2| \leq \log \left(D^{1 / 4} / 2\right)$ the vector $v_{t}=\left(D^{-1 / 4}, D^{-1 / 4}\right) h_{t}^{-1}$ would still be of norm less than one. Note that $v_{t}$ is moving along a hyperbola for varying values of $t$. The unimodular lattice $\Lambda_{\mathscr{O}}^{1} h_{t}^{-1}$ can only contain (up to sign) one vector of length less than one. Therefore, $t \mapsto \Lambda_{\mathscr{O}}^{1} h_{t}^{-1}$ is injective for $t \in\left[-\frac{1}{2} \log D+\right.$ $\left.2 \log 2, \frac{1}{2} \log D-2 \log 2\right]$ and the lemma follows.

We recall the notion of topological entropy for a continuous transformation $T$ : $Y \rightarrow Y$ on a compact metric space $(Y, d)$. For $\eta>0$ and a positive integer $N$ let $s_{\eta, N}(\beta)$ be the maximal number of points $y_{1}, y_{2}, \ldots, y_{s_{\eta, N}}$ such that for any $i \neq j$ there exists some $0 \leq k<N$ with $d\left(T^{k} y_{i}, T^{k} y_{j}\right)>\eta$. Then the topological entropy is defined by

$$
\mathrm{h}_{\mathrm{top}}(T)=\lim _{\eta \rightarrow 0} \limsup _{n \rightarrow \infty} \frac{\log s_{\eta, n}(\beta)}{n} .
$$

The following is a simple special case of a very general fact, see [23] and the references therein.

Lemma 7.3. For any $\epsilon>0$ there exists $\delta>0$ such that the restriction $T$ of (the time one map of the geodesic flow) $h_{1}$ to

$$
Y_{\delta}=\left\{x \in \Omega_{\delta}^{\prime}: h_{t} . x \in \Omega_{\delta}^{\prime} \text { for all } t>0\right\}
$$

satisfies $\mathrm{h}_{\mathrm{top}}(T)>1-\epsilon$. In fact, we have $s_{\eta, N} \geq e^{(1-\epsilon) N}$ for sufficiently small $\eta$.

Proof. Recall that $u \in \mathbb{R}$ is badly approximable $(B A)$ if $^{26} \liminf _{m \rightarrow \infty} m\langle m u\rangle>0$ and that the set of badly approximable numbers has Hausdorff dimension one. Moreover, since the Hausdorff dimension of a countable union is a supremum of the dimensions of the individual sets, it follows that for small enough $\delta>0$ the set $B A(\delta)$ of elements $u \in\left[0, \frac{1}{2}\right]$ with $\liminf _{m \rightarrow \infty} m\langle m u\rangle>\delta$ has Hausdorff dimension greater than $1-\epsilon$. In particular, for sufficiently small $\eta_{1}>0$ there exist at least $\eta_{1}^{-(1-\epsilon)}$ many such $u \in B A(\delta)$ that are at least $\eta_{1}$ far apart.

Let $x_{u}=\Gamma\left(\begin{array}{cc}1 & -u \\ 1\end{array}\right)$ for some $u \in B A(\delta)$. We claim that $x_{u} \in Y_{\delta}$. For, if not, there exists $t>0$ is sothat the lattice $\mathbb{Z}^{2}\left(\begin{array}{cc}1 & -u \\ & 1\end{array}\right)\left(\begin{array}{ll}e^{-t / 2} & \\ & e^{t / 2}\end{array}\right)$ contains a vector $\left(m e^{-t / 2},\left|m u+m^{\prime}\right| e^{t / 2}\right)$ whose coordinates have product $\leq \delta$ in absolute value. Then $m \neq 0$ and $\left|m\left(m u+m^{\prime}\right)\right|<\delta$.

Since $h_{1}\left(\begin{array}{ll}1 & u \\ & 1\end{array}\right) h_{1}^{-1}=\left(\begin{array}{cc}1 & e u \\ & 1\end{array}\right)$ it follows that the distance between two close by points on the same orbit of $\left(\begin{array}{cc}1 & \mathbb{R} \\ & 1\end{array}\right)$ is uniformly increasing until the distance is bigger than the injectivity radius of the local isomorphism between $G$ and $X$ at the image point. For $u_{1}, u_{2} \in B A(\delta)$ the points $x_{i}=\Gamma\left(\begin{array}{cc}1 & -u_{i} \\ 1\end{array}\right)$ will stay forever in $\Omega_{\delta}^{\prime}$. So if $u_{1}, u_{2}$ are $\eta_{1}=e^{-N} \eta_{0}$ far apart, we can ensure by choosing $\eta_{0}$ sufficiently small that $d\left(T^{k} \cdot x_{1}, T^{k} \cdot x_{2}\right)>\eta$ for some $k$ with $0 \leq k<N$. This shows that $s_{\eta, N} \geq e^{(1-\epsilon) N}$ for sufficiently large $N$ and so $\mathrm{h}_{\text {top }}(T) \geq 1-\epsilon$ as claimed.

\footnotetext{
${ }^{26}$ In this context, $\langle x\rangle$ denotes the nearest integer.
} 
To obtain periodic points from the above lemma regarding topological entropy we need the following standard facts from hyperbolic dynamics. We do not give here the statements in their full strength - not even for the special case considered here, see e.g. [22, Sec. 18.1.].

Lemma 7.4. (Shadowing lemma) For any $\eta_{s}>0$ there exists $\rho_{s}>0$ such that if $d\left(x_{-}, x_{+}\right)<\rho_{s}$, then there exists $y$ with $d\left(h_{t} . y, h_{t} . x_{-}\right)<\eta_{s}$ for all $t \leq 0$ and $d\left(h_{t} . y, h_{t} . x_{+}\right)<\eta_{s}$ for all $t \geq 0$.

(Anosov closing lemma) For any $\eta_{c}>0$ there exists $\rho_{c}>0$ such that if $d\left(x, h_{N} x\right)<$ $\rho_{c}$ for some $N \geq 1$, then there exists $y$ and $T \in\left[N-\eta_{c}, N+\eta_{c}\right]$ such that $h_{T} \cdot y=y$ and $d\left(h_{t} . x, h_{t} . y\right)<\eta_{c}$ for $t \in[0, N]$.

Proof. Write $x_{-}=g \cdot x_{+}$with $g=\left(\begin{array}{ll}g_{11} & g_{12} \\ g_{21} & g_{22}\end{array}\right), d(g, e)<\rho$ and $\operatorname{det} g=1$. Define $y=\left(\begin{array}{cc}1 & u \\ & 1\end{array}\right) \cdot x_{-}$. It is easy to see that for small enough values of $u$ this $y$ will always satisfy the first part of the shadowing statement. Since $y=\left(\begin{array}{ll}1 & u \\ & 1\end{array}\right)\left(\begin{array}{ll}g_{11} & g_{12} \\ g_{21} & g_{22}\end{array}\right) \cdot x_{+}=$ $\left(\begin{array}{cc}g_{11}+g_{21} u & g_{12}+g_{22} u \\ g_{21} & g_{22}\end{array}\right) \cdot x_{+}$, it follows similarly that for $u=-\frac{g_{12}}{g_{22}}$ both parts to shadowing hold true.

Suppose now $h_{N} \cdot x=g . x$ with $g$ as before. Define $\tilde{x}=\left(\begin{array}{ll}1 & \\ u & 1\end{array}\right) . x$. Then

$$
\begin{aligned}
h_{N} . \tilde{x} & =h_{N}\left(\begin{array}{ll}
1 & \\
u & 1
\end{array}\right) h_{N}^{-1} h_{N} \cdot x=\left(\begin{array}{cc}
1 & \\
e^{-N} u & 1
\end{array}\right) g\left(\begin{array}{cc}
1 & \\
-u & 1
\end{array}\right) \cdot \tilde{x} \\
& =\left(\begin{array}{cc}
g_{11}-u g_{12} & g_{12} \\
-e^{-N} g_{12} u^{2}+e^{-N} g_{11} u-g_{22} u+g_{21} & g_{22}+e^{-N} u g_{12}
\end{array}\right) \cdot \tilde{x} .
\end{aligned}
$$

If $\rho_{c}$ and so $\left(g_{11}-1\right), g_{12}, g_{21},\left(g_{22}-1\right)$ are sufficiently small, the quadratic polynomial in the low left corner has a unique root $u$ close to zero. In other words by replacing $x$ by the close by point $\tilde{x}$ we can assume that $g_{21}=0$. Now define $y=\left(\begin{array}{ll}1 & v \\ & 1\end{array}\right) . \tilde{x}$. A similar calculation (which in fact now leads to a linear equation) shows the Anosov closing lemma.

Lemma 7.5. Let $\epsilon$ and $\delta$ be as in Lemma 7.3. Then the number of periodic orbits of length less than $V$ that are contained in $\Omega_{\delta}^{\prime}$ is $\gg e^{(1-\epsilon) V}$.

Proof. By Lemma 7.3 we can choose $\eta$ such that for large enough $N$ there are $\gg$ $e^{(1-\epsilon) V}$ many points $x_{1}, \ldots \in Y_{\delta}$ with $d\left(h_{k} \cdot x_{i}, h_{k} \cdot x_{j}\right)>\eta$ for $0 \leq k=k(i, j)<N$. We are going to apply both parts of Lemma 7.4. The parameters $\eta_{s}$ and $\eta_{c}$ we choose such that after application of both statements to get points $y_{1}, \ldots \in X$ we still have $d\left(h_{k} . y_{i}, h_{k} . y_{j}\right)>\eta / 2$ for $k=k(i, j)$. By choosing the parameters for the closing statement first, we can assume that $2 \eta_{s}<\rho_{c}$. Since the geodesic flow is ergodic, there exists a finite $\rho_{s}$-dense set of points $z_{1}, \ldots, z_{\ell}$ in $\Omega_{\delta}^{\prime}$ and for every $z_{i}$ finitely many times $t(i, j)$ such that the set of points $h_{t(i, j)} . z_{i}$ for varying $j$ is $\left(\rho_{c}-2 \eta_{s}\right)$ dense in $\Omega_{\delta}^{\prime}$. Therefore, for every $x_{m}$ we can find a $z_{i}$ with $d\left(h_{N} \cdot x_{m}, z_{i}\right)<\rho_{s}$ and obtain by shadowing a point $\tilde{x}_{m}$ with $d\left(\tilde{x}_{m}, h_{N+t(i, j)} . \tilde{x}_{m}\right)<\rho_{c}$. Applying the closing lemma, we arrive at points $y_{1}, \ldots \in X$ with periodic orbits. These periodic points belong for varying $N$ to a fixed compact set, namely to a neighborhood of 
the union of $\Omega_{\delta}^{\prime}$ and the finite pieces of the orbits of $z_{1}, \ldots, z_{\ell}$ used in the argument. The length of the orbits are all below $V=N+\max _{i, j} t_{i, j}+\eta_{c}$. In particular, every periodic orbit can be counted at most $O(V)$ times since any two points in the list are at some moment $\eta / 2$ far apart. Since $\epsilon$ is arbitrary, this does not affect the statement of the lemma.

Proof of Theorem 7.1. Let $\epsilon, \delta$ be as in Lemma 7.3. Since there is a lower bound on the length of an $H$-orbit we have

$$
\sum_{\operatorname{vol}(\Gamma g H) \leq V, \Gamma g H \subset \Omega_{\delta}^{\prime}} \operatorname{vol}(\Gamma g H) \gg e^{(1-\epsilon) V}
$$

by Lemma 7.5. If we set $V=\log \Delta-C$, then $\operatorname{vol}(\Gamma g H) \leq V \operatorname{implies} \operatorname{disc}(\Gamma g H) \leq \Delta$ by Lemma 7.2. Therefore,

$$
\sum_{\operatorname{disc}(\Gamma g H) \leq \Delta, \Gamma g H \subset \Omega_{\delta}^{\prime}} \operatorname{vol}(\Gamma g H) \gg e^{(1-\epsilon) V} \gg \Delta^{1-\epsilon}
$$

as claimed.

7.2. Fields of small regulator and non-equidistributed orbits in higher rank; proof of Theorem 1.10. As an example which is illuminating in its own right, we construct using the above parameterizations a sequence of periodic $H$ orbits for which a positive fraction of mass escapes to the cusp. More precisely, we shall construct a sequence of periodic $H$-orbits $Y_{i}$ on $\mathrm{PGL}_{n}(\mathbb{Z}) \backslash \mathrm{PGL}_{n}(\mathbb{R})$ with the following properties, for some constants $a_{n}, b_{n}>0$ :

(1) The number of $Y_{i}$ with discriminant $\leq \Delta$ is $\gg \Delta^{a_{n}}$.

(2) Any weak limit of the $H$-invariant probability measures $\mu_{Y_{i}}$ on $Y_{i}$ has total mass $\leq 1-b_{n}$.

This example will, in particular, prove Theorem 1.10 asserted in the Introduction. We would like to thank Peter Sarnak for suggesting the possibility of it.

Proof. Indeed, Duke has established (cf. [8, $\S 3$, Prop. 1]) that, for every degree $n$, there is a constant $C=C(n)$ and infinitely many totally real fields $K_{i}$ whose Galois closure has Galois group $S_{n}$ and whose regulators satisfy the inequality $R_{K_{i}}<C\left(\log \operatorname{disc}\left(K_{i}\right)\right)^{n-1}$.

For each such $K_{i}$, let $\mathscr{O}_{i}$ be the maximal order, fix an algebra isomorphism $K_{i} \otimes \mathbb{R} \rightarrow \mathbb{R}^{n}$, and consider the periodic orbit associated to the triple $\left(K_{i},\left[\mathscr{O}_{i}\right], \theta\right)$.

Let $\alpha: K_{i} \otimes \mathbb{R} \rightarrow \mathbb{R}^{n}$ be the map $x \mapsto\left(\log \left|\theta(x)_{1}\right|, \log \left|\theta(x)_{2}\right|, \ldots, \log \left|\theta(x)_{n}\right|\right)$. Let $\Lambda$ be the image of $\mathscr{O}_{i}^{\times}$under the map $\alpha$. Then the $\mathbb{R}$-span $\Lambda_{\mathbb{R}}$ of $\Lambda$ is, by Dirichlet's unit theorem, precisely the subspace $\left\{\left(y_{1}, \ldots, y_{n}\right) \in \mathbb{R}^{n}: \sum y_{i}=0\right\}$, the image under $\alpha$ of elements $\left(K_{i} \otimes \mathbb{R}\right)^{(1)}$ of norm 1. Moreover, $\alpha$ induces an isomorphism of torii

$$
\alpha:\left(K_{i} \otimes \mathbb{R}\right)^{(1)} / \mathscr{O}_{i}^{\times} \rightarrow \Lambda_{\mathbb{R}} / \Lambda
$$

Endowing the left-hand torus with the measure transported from Lebesgue measure on $\Lambda_{\mathbb{R}} \subset \mathbb{R}^{n}$, the volume of the left-hand torus is $R_{K_{i}}$. On the other hand:

(1) Since $K_{i}$ has no intermediate subfields, all nonzero elements of $\Lambda$ have Euclidean length $\geq c \log \operatorname{disc}\left(K_{i}\right)$, for a certain constant $c$ depending only on $n$ (cf. e.g. 40] or proof of Corollary 4.2 (ii)) 
(2) If $x \in\left(K_{i} \otimes \mathbb{R}\right)^{(1)}$ is such that $|\log | \theta(x)_{i}|| \leq \frac{c}{2} \log \operatorname{disc}\left(K_{i}\right)$ for each $i$, then the lattice $\theta\left(x \cdot \mathscr{O}_{i}\right)$, which has covolume $\asymp \operatorname{disc}(K)^{1 / 2}$, contains a vector of Euclidean length $\leq \sqrt{n}\left(\operatorname{disc} K_{i}\right)^{c / 2}$.

Fix a compact subset $\Omega \subset \mathrm{PGL}_{n}(\mathbb{Z}) \backslash \mathrm{PGL}_{n}(\mathbb{R})$. It follows from these remarks that there is a subset $\left(K_{i} \otimes \mathbb{R}\right)^{(1)} / \mathscr{O}_{i}^{\times}$of volume $\gg c^{\prime}\left(\log \operatorname{disc}\left(K_{i}\right)\right)^{n-1}$, where $c^{\prime}$ depends only on $n$, such that the lattice $\theta\left(x \cdot \mathscr{O}_{i}\right)$ does not belong to $\Omega$ for all sufficiently large $i$. Since the total volume of $\left(K_{i} \otimes \mathbb{R}\right)^{(1)} / \mathscr{O}_{i}^{\times}$w.r.t. the measure defined above was $R_{K_{i}}<C\left(\log \operatorname{disc}\left(K_{i}\right)\right)^{n-1}$, this implies the conclusion.

\section{REFERENCES}

[1] Yves Benoist and Hee Oh. Equidistribution of rational matrices in their conjugacy classes. to appear in GAFA.

[2] Armand Borel and Harish-Chandra. Arithmetic subgroups of algebraic groups. Ann. of Math. (2), 75:485-535, 1962.

[3] J. Bourgain, A. Glibichuk, and S. Konyagin. Estimate for the number of sums and products and for exponential sums in fields of prime order. Preprint, 2005.

[4] Jean Bourgain and Mei-Chu Chang. Sum-product theorem and exponential sum estimates in residue classes with modulus involving few prime factors. C. R. Math. Acad. Sci. Paris, 339(7):463-466, 2004.

[5] J. W. S. Cassels and H. P. F. Swinnerton-Dyer. On the product of three homogeneous linear forms and the indefinite ternary quadratic forms. Philos. Trans. Roy. Soc. London. Ser. A., 248:73-96, 1955.

[6] S. G. Dani and G. A. Margulis. Limit distributions of orbits of unipotent flows and values of quadratic forms. In I. M. Gel' fand Seminar, volume 16 of Adv. Soviet Math., pages 91-137. Amer. Math. Soc., Providence, RI, 1993.

[7] W. Duke. Hyperbolic distribution problems and half-integral weight Maass forms. Invent. Math., 92(1):73-90, 1988.

[8] W. Duke. Extreme values of Artin $L$-functions and class numbers. Compositio Math., 136(1):103-115, 2003.

[9] M. Einsiedler and A. Katok. Invariant measures on $G / \Gamma$ for split simple Lie-groups $G$. Comm. Pure Appl. Math., 56(8):1184-1221, 2003.

[10] M. Einsiedler and A. Katok. Rigidity of measures - the high entropy case and non-commuting foliations. Israel J. Math., 148:169-238, 2005. Probability in mathematics.

[11] M. Einsiedler, A. Katok, and E. Lindenstrauss. Invariant measures and the set of exceptions to Littlewood's conjecture. to appear in Ann. of Math.

[12] Manfred Einsiedler and Elon Lindenstrauss. Diagonal flows on locally homogeneous spaces and number theory. to appear in the Proceedings of the International Congress of Mathematicians 2006 (29 pages), 2006.

[13] Manfred Einsiedler, Elon Lindenstrauss, Philippe Michel, and Akshay Venkatesh. The distribution of periodic torus orbits, II. in preparation, 2006.

[14] Manfred Einsiedler, Elon Lindenstrauss, Philippe Michel, and Akshay Venkatesh. The distribution of periodic torus orbits, III: Duke's theorem for cubic fields. in preparation, 2006.

[15] Alex Eskin and Hee Oh. Representations of integers by an invariant polynomial and unipotent flows. to appear in Duke Math. Journal.

[16] Alexander Gorodnik. Open problems from the workshop "Emerging Applications of Measure Rigidity" held at the American Institute of Mathematics. 2004. Available at http://www.aimath.org/WWN/measrigid.

[17] Gergely Harcos and Philippe Michel. The subconvexity problem for Rankin-Selberg Lfunctions and equidistribution of Heegner points. II. Invent. Math., 163(3):581-655, 2006.

[18] Thomas W. Hungerford. Algebra, volume 73 of Graduate Texts in Mathematics. SpringerVerlag, New York, 1980. Reprint of the 1974 original.

[19] Boris Kalinin and Ralf Spatzier. Rigidity of the measurable structure for algebraic actions of higher-rank Abelian groups. Ergodic Theory Dynam. Systems, 25(1):175-200, 2005.

[20] A. Katok and R. J. Spatzier. Invariant measures for higher-rank hyperbolic abelian actions. Ergodic Theory Dynam. Systems, 16(4):751-778, 1996. 
[21] A. Katok and R. J. Spatzier. Corrections to: "Invariant measures for higher-rank hyperbolic abelian actions" [Ergodic Theory Dynam. Systems 16 (1996), no. 4, 751-778; MR 97d:58116]. Ergodic Theory Dynam. Systems, 18(2):503-507, 1998.

[22] Anatole Katok and Boris Hasselblatt. Introduction to the modern theory of dynamical systems, volume 54 of Encyclopedia of Mathematics and its Applications. Cambridge University Press, Cambridge, 1995. With a supplementary chapter by Katok and Leonardo Mendoza.

[23] D. Y. Kleinbock and G. A. Margulis. Bounded orbits of nonquasiunipotent flows on homogeneous spaces. In Sinaй's Moscow Seminar on Dynamical Systems, volume 171 of Amer. Math. Soc. Transl. Ser. 2, pages 141-172. Amer. Math. Soc., Providence, RI, 1996.

[24] Elon Lindenstrauss. Invariant measures and arithmetic quantum unique ergodicity. Ann. of Math. (2), 163(1):165-219, 2006.

[25] Elon Lindenstrauss and Barak Weiss. On sets invariant under the action of the diagonal group. Ergodic Theory Dynam. Systems, 21(5):1481-1500, 2001.

[26] Yu. V. Linnik. Ergodic properties of algebraic fields. Translated from the Russian by M. S. Keane. Ergebnisse der Mathematik und ihrer Grenzgebiete, Band 45. Springer-Verlag New York Inc., New York, 1968.

[27] G. A. Margulis. Oppenheim conjecture. In Fields Medallists' lectures, volume 5 of World Sci. Ser. 20th Century Math., pages 272-327. World Sci. Publishing, River Edge, NJ, 1997.

[28] G. A. Margulis and G. M. Tomanov. Invariant measures for actions of unipotent groups over local fields on homogeneous spaces. Invent. Math., 116(1-3):347-392, 1994.

[29] G. A. Margulis and G. M. Tomanov. Measure rigidity for almost linear groups and its applications. J. Anal. Math., 69:25-54, 1996.

[30] Gregory Margulis. Problems and conjectures in rigidity theory. In Mathematics: frontiers and perspectives, pages 161-174. Amer. Math. Soc., Providence, RI, 2000.

[31] Shahar Mozes and Nimish Shah. On the space of ergodic invariant measures of unipotent flows. Ergodic Theory Dynam. Systems, 15(1):149-159, 1995.

[32] Hee Oh. Finiteness of compact maximal flats of bounded volume. Ergodic Theory Dynam. Systems, 24(1):217-225, 2004.

[33] Vladimir Platonov and Andrei Rapinchuk. Algebraic groups and number theory, volume 139 of Pure and Applied Mathematics. Academic Press Inc., Boston, MA, 1994. Translated from the 1991 Russian original by Rachel Rowen.

[34] Alexandru Popa. Central values of rankin l-series over real quadratic fields. to appear in Compositio Math.

[35] Gopal Prasad and M. S. Raghunathan. Cartan subgroups and lattices in semi-simple groups. Ann. of Math. (2), 96:296-317, 1972.

[36] M. S. Raghunathan. Discrete subgroups of Lie groups. Springer-Verlag, New York, 1972. Ergebnisse der Mathematik und ihrer Grenzgebiete, Band 68.

[37] Marina Ratner. On Raghunathan's measure conjecture. Ann. of Math. (2), 134(3):545-607, 1991.

[38] Marina Ratner. Raghunathan's topological conjecture and distributions of unipotent flows. Duke Math. J., 63(1):235-280, 1991.

[39] M. Rees. Some $R^{2}$-anosov flows. 1982.

[40] Robert Remak. Über Grössenbeziehungen zwischen Diskriminante und Regulator eines algebraischen Zahlkörpers. Compositio Math., 10:245-285, 1952.

[41] Daniel J. Rudolph. $\times 2$ and $\times 3$ invariant measures and entropy. Ergodic Theory Dynam. Systems, 10(2):395-406, 1990.

[42] Peter Sarnak. Reciprocal geodesics. preprint (25 pages)., 2006.

[43] Nimish A. Shah. Uniformly distributed orbits of certain flows on homogeneous spaces. Math. Ann., 289(2):315-334, 1991.

[44] Carl Ludwig Siegel. The average measure of quadratic forms with given determinant and signature. Ann. of Math. (2), 45:667-685, 1944.

[45] George Tomanov. Actions of maximal tori on homogeneous spaces. In Rigidity in dynamics and geometry (Cambridge, 2000), pages 407-424. Springer, Berlin, 2002.

[46] George Tomanov and Barak Weiss. Closed orbits for actions of maximal tori on homogeneous spaces. Duke Math. J., 119(2):367-392, 2003. 